\title{
Particle-resolved PIV experiments of solid-liquid mixing in a turbulent stirred tank
}

\author{
Genghong Li, Zhengming Gao, Zhipeng Li, Jiawei Wang \\ Beijing Advanced Innovation Center for Soft Matter Science and Engineering, Beijing University of Chemical Technology, Beijing 100029, China \\ State Key Laboratory of Chemical Resource Engineering, School of Chemical Engineering, Beijing University of Chemical Technology, Beijing 100029, China \\ J.J. Derksen \\ School of Engineering, University of Aberdeen, Aberdeen AB24 3UE, UK \\ Correspondence concerning this article should be addressed to Zhipeng Li at lizp@mail.buct.edu.cn \\ AIChE Journal - Accepted - August 2017
}

\begin{abstract}
Particle Image Velocimetry (PIV) experiments on turbulent solid-liquid stirred tank flow with careful refractive index matching of the two phases have been performed. The spatial resolution of the PIV data is finer than the size of the spherical, uniformly sized solid particles, thereby providing insight in the flow around individual particles. The impeller is a down-pumping pitch-blade turbine. The impeller-based Reynolds number has been fixed to $\mathrm{Re}=10^{4}$. Overall solids volume fractions up to $8 \%$ have been investigated. The PIV experiments are impeller-angle resolved, i.e. conditioned on the angular position of the impeller. The two-phase systems are in partially suspended states with an inhomogeneous distribution of solids: high solids loadings near the bottom and near the outer walls of the tank, much less solids in the bulk of the tank. The liquid velocity fields show very strong phase coupling effects with the particles increasingly attenuating the overall circulation patterns as well as the liquid velocity fluctuation levels when the solids volume fraction is increased.
\end{abstract}

Keywords: solid-liquid suspension; particle image velocimetry; refractive index matching; stirred tank; high-resolution flow measurement 


\section{Introduction}

Solid-liquid stirred tanks are widely used in many industrial processes for production of chemicals, foods and pharmaceuticals; they are applied for industrial crystallization processes, metallurgical applications, as catalytic slurry reactors, and in water treatment. In the operating units, the rates of momentum, mass and heat transfer, and chemical reactions are greatly affected by the solid-liquid two-phase turbulent flow conditions. This makes it very relevant to investigate turbulent structures and characteristics in solid-liquid stirred tanks.

There is extensive literature in this field, on the experimental side, as well as on the computational side. Based on extensive sets of experimental observations, Zwietering ${ }^{1}$ introduced the just-suspended speed $\left(N_{\mathrm{js}}\right)$ in solid-liquid stirred tanks as the impeller speed when no particles remain still on the bottom of the tank for more than $1-2 \mathrm{~s}$. With this criterion, many other researchers ${ }^{2-5}$ measured $N_{\mathrm{js}}$ in a great variety of mixing configurations. This was summarized by Kasat et al. ${ }^{6}$ in a review paper. Where $N_{\mathrm{js}}$ is a global system characteristic, various experimental methods have been used to provide a local view of solids suspension processes. Hosseini et al. ${ }^{7}$ used an Electrical Resistance Tomography (ERT) technique to measure solid concentration distributions and investigate how the impeller type, impeller speed, impeller off-bottom clearance and particle size affected mixing efficiency. Carletti et al. ${ }^{8}$ also used ERT to investigate the spatial solids distribution in a stirred tank and they proposed a method to evaluate the solids distribution under different working conditions and geometrical set-up. Tahvildarian et al. ${ }^{9}$ and Harrison et al. ${ }^{10}$ used ERT to study how the working condition, particle size and particle concentration affected the solids concentration homogeneity in a stirred tank. Positron Emission Particle Tracking is a non-intrusive Lagrangian flow visualization technique that has been used to obtain the full 3D velocity and concentration fields of both the continuous phase and the dispersed phase in opaque mixing equipment. ${ }^{11,12}$ Computer Automated Radioactive Particle Tracking is another non-intrusive technique that has been used to measure the time-averaged velocities and turbulent kinetic energy (TKE) of the solids as well as the solids sojourn time distribution. ${ }^{13}$ In addition, a review focused on different kinds of measurement techniques about particle concentrations in the solid-liquid stirred tanks has been performed by Tamburini et $\mathrm{al}^{14}$. An important parameter to characterize solids distribution is the cloud height. It is the vertical location of the interface between clear liquid and particle-laden liquid. ${ }^{15}$ Sardeshpande et al. ${ }^{16}$ used an ultrasound velocity profiler to study how the impeller speed affected hysteresis in cloud height and compared results with those of computational fluid dynamics simulations. 
As for investigations about the flow field, laser-based optical imaging techniques such as Laser Doppler Anemometry $(\text { LDA })^{17-19}$ and Particle Image Velocimetry (PIV) $)^{20-23}$ are the most widely used. In applying these techniques to solid-liquid systems, a main limiting factor is the solids concentration which - in the studies referred to here - was less than $1 \%$ by volume. ${ }^{17,21,23}$ The reason for this is that the laser light will get more and more obstructed and scattered by particles if we increase the solids volume fraction. This leads to erroneous velocity measurements and poor signal-to-noise ratios. In order to overcome this problem, researchers employed a Refractive Index Matching (RIM) method. ${ }^{24,25}$ In terms of applying the RIM method in solid-liquid stirred tanks, Micheletti et al. ${ }^{18}$ used a mixture of 1-methylnaphtalene, 1-chloronaphtalene and tetraline as the continuous phase and polystyrene/1 per cent divinylbenzene impurity-free monodisperse particles as the dispersed phase to study the liquid velocity characteristics in the presence of particles with solids volume fraction up to $2 \%$ by LDA. Virdung et al. ${ }^{19}$ used a mixture of benzyl alcohol/ethanol and glass spheres as the continuous and dispersed phases respectively to investigate the axial velocities and turbulence levels in a stirred tank by LDA with a maximum solids volume fraction up to $9 \%$. With the same continuous and dispersed phases, Virdung et al. ${ }^{20}$ also measured the velocities of both phases up to $1.5 \%$ solids by volume by using PIV combined with image analysis to separate the particles and liquid in their camera frames. Gabriele et al. ${ }^{22}$ used p-cymene and PMMA spheres as the continuous and dispersed phases respectively to investigate the turbulence properties of the fluid in a high throughput stirred vessel for both up-pumping and down-pumping configurations by PIV with up to $5 \%$ (by volume) particles. Kohnen et al. ${ }^{26}$ used a mixture of oil (Pharma 5 , DEA) and a light protective liquid (Eusolex, Merck) to match the refractive index of the particles (Duran glass). The solids volume fraction was up to $15 \%$ in this LDA experiment. In this way, Kohnen et al found that the TKE increased in the areas near the impeller and decreased in the remaining areas with the rise of solids fractions. However, the size of the particles as well as the resolution of velocity vectors in most investigations are less than or equal to one millimeter ${ }^{20,21,23,26}$, which means that the flow field around the particles is not resolved by the experiments. In the study by Gabriele et al. ${ }^{22}$, the diameter of the particles was 1.5 mm whereas the velocity vectors resolution was $171 \mu \mathrm{m}$ with the minimum interrogation area of $16 \times 16$ pixels and $50 \%$ overlap. As a result, there are about eight vectors over the diameter of the particles.

An important purpose of experimental studies on solid-liquid flows often is to provide validation data for computational simulations. The more details contained in the experimental data, the more critical simulation results can be assessed. In this respect it is relevant to note the trend in computational work towards particle-resolved simulations ${ }^{27,28}$ in which the flow around individual particles is calculated and used to determine - via computing forces and torques on the particles - the dynamics of the particles concurrently with the fluid flow. Major reasons for aspiring this level of details in simulations are to 
increase their predictive capabilities (such simulations largely forego the need for semi-empirical input for e.g. the drag force) and to facilitate studying phenomena at the solid-liquid interfaces, such as mass transfer or surface reactions. ${ }^{29,30}$ For validation purposes, we seek comparable resolution in experiments as in simulations. We therefore are looking for experimentally resolving the flow around individual particles suspended in a turbulently agitated tank under moderate to high solids loading conditions. As noted above, high resolution can be obtained by means of optical methods (LDA, PIV) provided optical accessibility can be achieved through matching the refractive index of the disperse and continuous phases. In this paper, we present a PIV study of an agitated, dense solid-liquid suspension where we have carefully matched the refractive index of spherical glass beads and the surrounding liquid. This makes the contents of the mixing tank transparent and accessible for laser light and cameras throughout its entire volume.

The PIV setup we have been using has a spatial resolution of the order of one velocity vector per millimeter. In order to have sub-particle PIV resolution we have used $d=8 \mathrm{~mm}$ sized spherical particles. These are large particles if compared to the applications we mentioned above; in e.g. crystallization or slurry reactors particles are hardly ever larger than $1 \mathrm{~mm}$. We have made sure, however, that in dimensionless terms we cover practically relevant process conditions. A major dimensionless number in suspension dynamics is the Archimedes number: $\operatorname{Ar} \equiv \frac{g \Delta \rho d^{3}}{\rho v^{2}}$ with $g$ gravitational acceleration, $\Delta \rho=\rho_{s}-\rho$ the density difference between solid and liquid, and $v$ the kinematic viscosity of the liquid. The Archimedes number can be interpreted as a measure for the square of the Reynolds number for a settling particle. By choosing $v$ to be approximately 20 times the viscosity of water (and with $d=8 \mathrm{~mm}$ as mentioned above) our experiments have an Archimedes number comparable to that of $1 \mathrm{~mm}$ particles in a watery liquid. In the same spirit, we have chosen impeller speeds to reach practically relevant values for the modified Shields number $\theta \equiv \frac{\rho N^{2} D^{2}}{g d \Delta \rho}$ that was recently identified as a determinant dimensionless number for levels of solids suspension in mixing tanks. ${ }^{27}$ In the expression for the Shields number $N$ and $D$ are the impeller speed (in $\mathrm{rev} / \mathrm{s})$ and impeller diameter respectively.

The aim of this paper is in the first place to show the feasibility of particle-resolved PIV experiments under mildly turbulent conditions and with appreciable solids loadings. Tank-averaged loadings are up to $8 \%$ by volume; locally solids volume fractions going beyond $30 \%$ were measured. In the second place, by comparing average flow structures as measured in single-phase systems by their multiphase counterparts we demonstrate the effect of adding solids on the flow dynamics (average velocity fields, turbulent fluctuation levels) in the mixing tank. In the third place, the flow system is designed such 
that it allows for one-on-one comparison with particle-resolved numerical simulations and thus provides an experimental data base for validation of computational approaches.

The paper is organized in the following manner. In the next section the experimental setup is discussed: definition of the flow system, PIV instrumentation, and image analysis. In the subsequent Results section we first provide PIV snapshots in which single-phase and particle-laden systems are compared. Secondly, we discuss the effect of the overall solid loading on the average distribution of solids over the tank, resolved for the impeller angle. Thirdly, averaged liquid velocity data and liquid velocity fluctuation levels are presented with again the averages conditioned on the impeller angle with an emphasis on phase-coupling effects. The final section provides a summary and conclusions.

\section{Experimental setup}

\section{Flow geometry}

The experiments were carried out in a rectangular stirred tank with a flat square base (side length $T=220 \mathrm{~mm}$ ) and height $300 \mathrm{~mm}$. The tank was made of tempered glass. A Perspex lid covered the liquid at height $H=T$ to avoid air entrainment and to provide a simple, no-slip condition in case one wants to mimic the flow by numerical simulations. A $45^{\circ}$ pitched-blade turbine of down-pumping configuration of diameter $D=158 \mathrm{~mm}$ was used as the agitator. It was placed at an off-bottom clearance $C=44 \mathrm{~mm}(C=T / 5)$. The main geometrical features of the stirred tank are shown in Figure 1 . The measurement plane was between $0<z / H<0.5$ and $0<x / T<0.5$ which is shown as the blue frame in Figure 1 . The angle $\theta$ is the angle between the impeller blade and the measurement plane, in the wake of the blade.

\section{Liquid and particles: refractive index matching}

In order to gain optical access to the area of interest and measure liquid velocities in the dense solid-liquid suspension system with an overall solids volume fraction up to $8 \%$, the refractive index (RI) $n$ of the liquid and solid should be accurately matched. Phenyl silicone oil $\left(n=1.4610\right.$ at $\left.21^{\circ} \mathrm{C}\right)$ was chosen as the continuous phase. Silica glass spheres $\left(n=1.460\right.$ at $\left.21^{\circ} \mathrm{C}\right)$ of average diameter $d=8 \mathrm{~mm}$ with a standard deviation of $\pm 0.2 \mathrm{~mm}$ (each sphere used was measured individually with an electronic vernier caliper) were used as the disperse phase. The properties of both the continuous and disperse phase at $21^{\circ} \mathrm{C}$ are shown in Table 1. 
Refractive indices were measured with an Abbe refractometer (WYA-2W, China) under well-controlled thermal conditions at the sodium $D$ line (wavelength $\lambda=589.3 \mathrm{~nm}$ ). The PIV experiments make use of laser light with $\lambda=532 \mathrm{~nm}$. The refractive index somewhat depends on the wavelength. As an example, for silica glass at $20^{\circ} \mathrm{C}, n=1.458$ for $\lambda=589.3 \mathrm{~nm}$, and $n=1.461$ for $\lambda=532 \mathrm{~nm}^{31}$

Figure 2 illustrates the sensitivity of refractive index for the levels of optical distortion: a change of $n$ by less than 0.01 makes a significant difference in this respect. This makes it necessary to control the temperature in the experiments since the $\mathrm{RI}$ of most liquids and solids decreases with increasing temperature with the RI of liquids being more sensitive to temperature. For the working liquid, the relationship between refractive index and temperature as measured with the Abbe refractometer is shown in Figure 3a. As we can see, the RI of phenyl silicone oil is relatively insensitive to the temperature (a decline of 0.0022 with a rise of $\left.5^{\circ} \mathrm{C}\right)$. The temperature in the experiments was controlled to within $\pm 1^{\circ} \mathrm{C}$ so that the RI only varies slightly with little effect on the accuracy of the experiments.

Although the conditions in the experiments were carefully controlled in order to achieve high levels of refractive index matching, it is virtually impossible to attain complete matching. Zachos et al. ${ }^{32}$ claimed that this was due to inhomogeneity of the glass material and imperfect surface finishing of the spheres. Additionally, there are minute bubbles in the glass spheres which might affect the measurement of the liquid phase velocities. ${ }^{33}$ At the same time, the surface imperfections are beneficial as they help in identifying sphere cross sections in the PIV images.

The way the viscosity of the working fluid depends on the temperature is shown in Figure 3b. These data were obtained with a MARS40 Rheometer (Haake, Germany). At the reference temperature of $21^{\circ} \mathrm{C}, \mu=0.02087 \mathrm{~Pa} \cdot \mathrm{s}$.

The stirred tank in operation with $8 \%$ solids is shown in Figure 4 a (with silica glass spheres); the impeller tip region is magnified in Figure 4c. A ruler is placed behind the tank to show the levels of transparency and distortion of light paths traversing the entire width of the tank. For comparison we show the same tank operating at the same Shields number with soda-lime glass spheres (Figures $4 \mathrm{~b}$ and $4 \mathrm{~d}$ ) that have a RI of 1.530 (silica glass has 1.460 , see Table 1 ). In the latter case, one can hardly see the ruler placed at the opposite side of the tank.

\section{Operating conditions}

The solids volume fractions in the experiments were set at $0 \%, 1 \%, 3 \%, 5 \%$ and $8 \%$. The impeller speed was fixed at $N=496 \mathrm{rpm}=8.27 \mathrm{rev} / \mathrm{s}$. With a liquid kinematic viscosity of $\nu=\mu / \rho=2.1 \cdot 10^{-5} \mathrm{~m}^{2} / \mathrm{s}$ (see Table 1 ), the Reynolds number 
associated to the impeller motion is $\operatorname{Re} \equiv \frac{N D^{2}}{v}=9.8 \cdot 10^{3}$; this indicates mildly turbulent flow. ${ }^{34}$ The modified Shields number $\theta \equiv \frac{\rho N^{2} D^{2}}{g d \Delta \rho}=17.7$. Particle resolved simulations ${ }^{27}$ for similar flow systems show partial suspension conditions at this level of the Shields number. The combination of liquid and particles is characterized by an Archimedes number of $\operatorname{Ar} \equiv \frac{g \Delta \rho d^{3}}{\rho v^{2}}$ $=1.39 \cdot 10^{4}$. A Stokes number has been defined as $\mathrm{St} \equiv \frac{2}{9} \frac{\rho_{s}}{\rho} \frac{d^{2} 4 N}{v}$ where we take the inverse of the blade-passage frequency - $1 /(4 N)$ - as the time scale of the flow. In the current study St=50.0; particle inertia is thus anticipated to be important and collisions (particle-particle and particle-impeller) are expected to be intense.

The Zwietering correlation ${ }^{1}$ for the just-suspended impeller speed reads

$$
N_{j s}=s \frac{(d)^{0.2} v^{0.1}\left(100 \phi \rho_{s} / \rho\right)^{0.13}}{D^{0.85}}\left(\frac{g \Delta \rho}{\rho}\right)^{0.45}
$$

with $s$ a dimensionless parameter that depends on the flow configuration, $\phi$ the solids volume fraction, and $N_{\mathrm{j} s}$ in rev/s. If - as a coarse estimate - we take $s=5$ and apply the correlation to our highest solids loading of $\phi=0.08$, we find $N_{\mathrm{js}}=13.9 \mathrm{rev} / \mathrm{s}$. Our impeller speed of $N=8.27 \mathrm{rev} / \mathrm{s}$ is thus - at least for $8 \%$ solids loading - slightly below $N_{\mathrm{js}}$. Also in light of this metric we do not expect complete off-bottom solids suspension.

\section{PIV system}

A 2D-PIV system (TSI, USA) was used in the experiments. The system consists of a $532 \mathrm{~nm} 200 \mathrm{~mJ} \mathrm{Nd}$ :YAG dual pulse laser (model No: Vlite-200, Beamtech, China), whose light beam goes through a spherical lens and a concave cylindrical lens to form a laser sheet with thickness of $1 \mathrm{~mm}$ at the measurement plane, a $4008 \times 2672$ pixels charge coupled device (CCD) camera (model No: PowerView Plus 11M, TSI, USA), a synchronizer (model No: Laser Pulse 610035, TSI, USA) that is used to synchronize the image capture and the laser pulses, an encoder (model No: A02H, Kubler, Germany) and this way obtains impeller-angle-resolved measurements and a computer with the TSI INSIGHT 3G software (version: 9.1) which manages the process of image capture and post-processing. Hollow glass beads (TSI, USA) with diameters in the range of 8-12 $\mu \mathrm{m}$ and density of $1500 \mathrm{~kg} / \mathrm{m}^{3}$ were used as tracer particles. 
The measurement area was a vertical, rectangular plane passing through the center of the tank (see Figure 1). Given the location of the plane, the Cartesian coordinates $x, y$ and $z$ correspond to radial, tangential and axial direction, respectively. The center of the tank bottom was selected as the origin of the Cartesian coordinates (see Figure 1). The angle in the wake of the blade and the measurement plane was defined as $\theta$, as shown in Figure 1.

A total of 500 pairs of images were captured for each impeller angle and each level of overall solids volume fraction. The synchronizer was triggered once per revolution by the encoder mounted on the shaft. However, since one image is about 21 $\mathrm{MB}$ it would take about $1 \mathrm{~s}$ for the CCD camera to transfer a pair of images to the computer hard disk. Therefore the capturing frequency was once every nine impeller revolutions given the impeller speed of $N=8.27 \mathrm{rev} / \mathrm{s}$. The interrogation windows were $48 X 48$ pixels with $50 \%$ overlap. The resolution of the images was $40.80 \mu \mathrm{m} /$ pixel so that the PIV system provides the $x$ and $z$ components of velocity vectors on a square grid with a spacing of $0.98 \mathrm{~mm}$, i.e. there are approximately eight vectors per particle diameter. The Fast Fourier Transform algorithm was used to carry out the cross-correlation processing. In terms of the

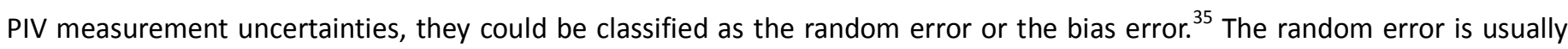
caused by the electrical noise in the camera and shot noise (independent from pixel to pixel and in time). Bias error mainly results from the image interrogation and the peak locking effect. The Nyquist Grid combined with the ZeroPad Mask method was used to interrogate the images in order to minimize the bias error. Peak locking is generally attributed to the under-resolved optical sampling of the particle images and relates to the choice of sub-pixel estimator. For the latter, the Gaussian sub-pixel estimator has been applied. This is considered a better choice for mitigating peak-locking effects. ${ }^{36}$ In relation to the former, a ratio between the tracer particle image diameter $\left(d_{\tau}\right)$ and the pixel spacing $\left(d_{r}\right)$ is usually used to identify the peak locking effect. Peak locking is dominant if $d_{\tau} / d_{r} \ll 1$. The $d_{\tau} / d_{r}$ in this PIV experiment is about 3 which is in the range of the optimum particle image diameter (2-3 pixels). ${ }^{37}$

\section{Image processing - detection of spheres}

A Matlab (version: R2015a, MathWorks, USA) code has been developed to detect the circumferences of the particles that are visible in the raw PIV images. This allows us to determine the solids distribution in the tank, as well as to eliminate the (unphysical) velocity vectors the PIV algorithm assigns to interrogation areas inside the particles.

The particle detection code operates as follows: first, we transform the original RGB images to gray images and then adjust the image intensity so as to increase the contrast with the Matlab function imadjust. We then apply a Circular Hough Transform (CHT) algorithm for finding circles in images as a means of detecting the circumferences of the particles. ${ }^{38}$ In this 
study, we used the Matlab function imfindcircles ${ }^{39}$ to perform the CHT. This function requires a number of input parameters: The Sensitivity factor was set to 0.925 , and the Edge gradient threshold to 0.01 . Furthermore, Object polarity was set to 'Bright' and Computation method to 'PhaseCode'. ${ }^{40}$ These settings were selected by applying imfindcircles to a set of PIV test images and comparing the particles detected by the algorithm with what we observe with the naked eye. Since the laser sheet has a thickness much smaller than the diameter of the particles, particle circumferences that show in the measurement plane should not overlap. This also is used to judge the performance of the particle detection algorithm. Sample testing results applied to a single PIV image are shown in Figure 5. We estimate that $5 \%$ of the particles in the images remain undetected and that approximately $10 \%$ of the particles are detected falsely. The majority of the latter is detected on the impeller and can be removed automatically in a subsequent Matlab step that keeps track of the way the impeller blades intersect with the image plane.

An original PIV RGB image is shown in Figure $6 a$ and the particle detection result is shown in Figure 6b. The velocity vectors calculated by the INSIGHT 3G software using the original PIV images are shown in Figure 6c. Subsequently, in Figure 6d the vectors inside the detected particles are removed. Figure $6 \mathrm{~d}$ illustrates the resolution of about eight vectors across the diameter of a sphere.

It is unfortunately not possible with the current setup to measure the velocities and rotation rates of the particles. A particle tracking approach based on two subsequent PIV realizations does not work since we only capture one instantaneous velocity field per nine impeller revolutions. Estimating particle displacement during the time between two frames forming a PIV image pair (60 $\mu \mathrm{s})$ cannot be done sufficiently accurately since this time is too short. For example, if a particle would move with a speed of $0.2 \mathrm{v}_{\text {tip }}(0.82 \mathrm{~m} / \mathrm{s})^{27}$ (where $\mathrm{v}_{\text {tip }}$ is the impeller tip speed $\mathrm{v}_{t i p}=\pi N D$ ), during this time the displacement is $49 \mu \mathrm{m}$. This is comparable to the accuracy with which particle locations have been determined in our Matlab routine which is approximately $41 \mu \mathrm{m}$ (one pixel on the PIV image). Therefore, this then gives us erratic and unreliable results for particle velocity.

\section{Analysis of flow properties}

Our experimental data consist of sets of 500 PIV realizations of the flow in the field of view identified in Figure 1 for tank-averaged solids volume fractions of $0 \%, 1 \%, 5 \%$ for impeller angles $\theta$ (see Figure 1 ) of $0^{\circ}, 30^{\circ}, 60^{\circ}$ and $3 \%, 8 \%$ for impeller angles $\theta$ of $60^{\circ}$ only, thus amounting to a total of 11 PIV data sets. 
The solids volume fraction distributions in the images were determined by assigning a ' 1 ' to pixels that fall inside a detected circle and ' 0 ' elsewhere. Averaging the pixel values over the 500 realizations then provides an estimate for the average local solids volume fraction field with sub-particle resolution.

The velocities averaged over the 500 PIV realizations are - by the nature of the experimental setup impeller-angle-resolved averages that we denote as $\bar{U}_{\theta}, \bar{V}_{\theta}, \bar{W}_{\theta}$ for the radial, tangential, and axial components respectively, with $\theta$ indicating the impeller angle. Velocity fluctuations are characterized by their root-mean-square (rms) values; e.g. $U_{\theta}^{\prime}=\sqrt{\overline{\left(U_{\theta}-\bar{U}_{\theta}\right)^{2}}}$. The turbulent kinetic energy $k$ relates to the rms velocity values: $k=\frac{1}{2}\left(U_{\theta}^{\prime 2}+V_{\theta}^{\prime 2}+W_{\theta}^{\prime 2}\right)$. Since we do not measure the tangential velocity component, the turbulent kinetic energy has been approximated as $k \approx \frac{3}{4}\left(U_{\theta}^{\prime 2}+W_{\theta}^{2}\right) \cdot{ }^{42}$ In presenting results, velocities have been scaled with the impeller tip speed $\mathrm{v}_{\text {tip }}$, and turbulent kinetic energy by $\mathrm{v}_{\text {tip }}^{2}$.

\section{Results and discussion}

\section{Instantaneous flow realizations - single-phase and solid-liquid flow}

We first will be showing single realizations of the flow field, with and without particles. For this, the overall measurement plane has been divided in seven sub-panels as indicated in Figure 7. The single-phase version of Panel (a) clearly shows the strong vortex that is shed from the impeller, where we note that this image was taken $60^{\circ}$ in the wake of an impeller blade. The corresponding liquid-solid image demonstrates that the presence of particles obstructs the formation of the vortex. This image again emphasizes the sub-particle resolution of the PIV measurements. Panels (b), (c) and (d) show the bottom region of the tank. As anticipated by the Shields number value of 17.7 and $N_{\mathrm{js}}$ estimate, we are dealing with a partially suspended solid-liquid system that has high solids concentrations near the bottom. The particles clearly hinder the liquid flow. This is most clear in the bottom-corner Panel (d) that for single-phase sees the impeller outstream impacting on the outer wall of the tank. The flow in solid-liquid Panel (d) is very much weaker. Panels (e), (f) and (g) are above the impeller where there is downflow near the shaft in the single as well as the two-phase system. Particles do get suspended to these vertical levels as shown in Panels (f) and (g).

In Figure 8 we quantitatively visualize the overall damping of the liquid flow by the particles by providing velocity magnitude data for the entire field of view. A much weaker flow is coming off the impeller in the two-phase system. This has 
consequences for the overall recirculation pattern in the tank and thus for the levels at which solids are being suspended. Hence, we observe a very strong two-way coupling between solids and liquid dynamics, at least for a tank-averaged solids volume fraction of $8 \%$.

\section{Averaged solids volume fraction}

We now turn to showing the spatial distributions of average quantities (solids volume fraction and velocity), based on the 500 single realizations as measured with PIV per experimental condition. Figures 9 and 10 show how the solids distribute themselves on average in the measurement plane (vertical plane through the center extending over $0<x / T<0.5$ and $0<z / H<0.5$ and $\mathrm{H}=\mathrm{T}$; see Figure 1).

As can be seen, the suspension process is only partial with particles collecting on the bottom underneath the impeller. For the lower overall solids volume fractions, the impeller stream is able to wash away particles near the bottom for $x>0.3 T$; at higher solids loadings this is less the case. The particles that get suspended to levels above the impeller hardly populate the center regions of the tank; solids distributions are thus very inhomogeneous.

In Figure 10 it shows that the impeller swept volume has relatively low solids concentration: the impeller is sweeping away the particles and the high inertia (Stokes number $\mathrm{St}=50$ ) of the particles makes that there is not sufficient time to fill this so created void until the next impeller blade comes by.

Vertical profiles of the solids concentration are given in Figures 11 and 12. As Figures 9 and 10, they reveal a strongly inhomogeneous distribution of solids. In Figure 11 at $\mathrm{x} / \mathrm{T}=0.20$ shows that there hardly are any particles above the impeller. There is a more even distribution of particles over the lower half of the tank height for $x / T=0.47$. The results show an interesting dependency of the vertical solids concentration with the impeller angle, see Figure 12 . At $x / T=0.47$ and near the bottom, peak solids volume fractions significantly depend on the angle $\theta$ of the impeller: particles settle in the corner of the tank when $\theta=0^{\circ}$ and are then partly swept away by the impeller stream which is felt when $\theta=30^{\circ}$ and even more so when $\theta=60^{\circ}$

The average vertical particle location in the entire field of view has been used to assess statistical convergence of the averaging process, see Figure 13. Calculating the average over an increasing number of PIV realizations shows stable results beyond averaging over approximately 300 realizations.

\section{Liquid phase velocities - the effect of solids}


As already observed in instantaneous realizations (e.g. in Figure 8), the liquid flow depends strongly on the presence of particles. In this section we aim at quantifying these effects; in the first place in terms of the impeller-angle-resolved average liquid velocities, in the second place in terms of velocity fluctuations levels and turbulent kinetic energy.

Figure 14 shows the average flow with increasing overall solids volume fraction (top to bottom) and increasing impeller angle (left to right). Two effects are immediately apparent: (1) the overall circulation patterns weaken with increasing numbers of particles in the tank; (2) the liquid flow near the bottom is attenuated if the solids volume fraction increases. As a way to quantify two-way coupling effects we present the downward velocity averaged over the horizontal dashed lines in the panels of Figure 14 at $\theta=0^{\circ}$ in Table 2. It shows that the downward velocity averaged over the horizontal lines ( $\left.U_{a v}\right)$, and thus the pumping capacity of the impeller, drops by nearly $30 \%$ when the solids volume fraction rises from $0 \%$ to $5 \%$.

The average velocity profiles in Figure 15 show the trends identified above in an alternative manner. The strength of the impeller outflow in radial direction (Figure 15a) monotonically decreases with increasing solids loading, with the strongest reduction taking place around the $3 \%$ (by volume) overall solids loading. The same panel also shows the weakening of the radial inward flow near the bottom due to the presence of many particles there. A consequence of the reduction of liquid circulation in the presence of particles is the reduction of the vertical velocities (in an absolute sense) on the horizontal line halfway the measurement plane (Figure 15b).

Finally we present velocity fluctuation levels where it should be reiterated that - since we perform impeller-angle-resolved measurements - the fluctuations observed are due to turbulence, not due to the periodic impeller motion. In the single-phase flow (Figure 16, upper row), turbulent fluctuations (with TKE as its measure) are mainly generated in the wake of the impeller blades. This turbulence hot-spot is then advected by the impeller stream in the radial directions and decays in strength. In a qualitative sense the same process is observed for the solid-liquid systems we investigated, see the remaining panels of Figure 16. The difference is that the peak levels of TKE get reduced significantly by the presence of particles. At $8 \%$ overall solids volume fraction the peak level in the contour plot of dimensionless TKE is 0.046 , in the single-phase case it is $\mathbf{0 . 0 6 5}$. The presence of particles weakening the turbulence intensities is in a good agreement with results of Unadkat et al. ${ }^{21}$ and Gabriele et al. ${ }^{22}$

Profiles of velocity fluctuation levels are shown in Figure 17. They consistently show the same trend of reduction in the presence of particles. The radial rms velocity has declined significantly when the solids volume fraction rises up to $3 \%$. When it goes up to $8 \%$ the average drop of radial rms velocity is about $20 \%$ in the region $0.1<\mathrm{z} / \mathrm{H}<0.5$ and the maximum drop exceeds 
$40 \%$ in the region $0<z / H<0.1$, see Figure 17 a. As for the axial rms velocity, the average decline is about $15 \%$ with the solids volume fractions rising to $8 \%$, see Figure $17 \mathrm{~b}$.

\section{Conclusions}

Refractive index matched PIV experiments on the turbulent solid-liquid flow in a stirred tank have been performed. The spatial resolution of the velocity data is an order of magnitude finer than the size of the - to a very good approximation - uniformly sized spherical particles. A critical step in the data analysis is the identification of the spheres that intersect with the laser sheet. A Matlab routine has been developed for this purpose, and its accuracy has been assessed as being of the order of $10 \%$. This means that the number of undetected and falsely detected particles is of the order of 0.1 times the number of detected particles. While the experiments are able to measure the velocity of the liquid with good resolution, as of now it is not possible to measure the instantaneous linear and/or angular velocities of the particles.

The single realizations of PIV data give a detailed insight in the flow around individual particles, as well as the way the liquid flow generated by the spinning impeller is altered by the presence of the particles. Strong damping effects due to the particles are observed. It is particularly interesting to see the formation of the very strong vortex in the wake of the impeller blade in single-phase flow being hindered by the presence of the particles.

We show that measuring 500 independent realizations of the flow leads to converged statistical quantities. Averaging over this many PIV realizations makes it possible to quantify the attenuation effects of the particles on the flow. As anticipated by the Shields number and the Zwietering correlation for the just-suspended impeller speed, the flow conditions are such that the particles are only partially suspended. As a result, the particles obstruct the flow near the bottom of the tank. This shifts the main recirculating pattern in the vertical field of view through the center of the tank in the upward direction. The particles in the volume above the impeller weaken this recirculation pattern.

All PIV experiments were conditioned upon the angle of the impeller (impeller-angle-resolved experiments). This makes that we are able to isolate the effect of turbulence in the velocity fluctuations. As for the average flow, also the turbulence intensities are attenuated strongly by the presence of particles. Peak levels of TKE - estimated based on two of the three fluctuating velocity component - show a reduction by about $41 \%$ when comparing single-phase flow with the flow volume containing $8 \%$ solids by volume. 
The experimental data form a detailed data set that can be used to directly compare with simulation results. Performing simulations that resolve the flow around individual of the current system thus is one of our future research lines. Improving the robustness of solid particle detection and - more importantly - determining the linear and angular velocity of the particles concurrently with liquid velocities is proposed as another important next step in our investigations.

\section{Acknowledgement}

The authors gratefully acknowledge the financial support from the National Natural Science Foundation of China (No.21376016).

\section{Literature cited}

1. Zwietering TN. Suspending of solid particles in liquid by agitators. Chem Eng Sci. 1958;8:244-253.

2. Nienow AW. Suspension of solid particles in turbine agitated baffled vessels. Chem Eng Sci. 1968;23:1453-1459.

3. Baldi G, Conti R, Alaria E. Complete suspension of particles in mechanically agitated vessels. Chem Eng Sci. 1978;33:21-25.

4. Davies JT. Particle suspension and mass transfer rates in agitated vessels. Chem Eng Process: Process Intensification. 1986;20:175-181.

5. Sharma R, Shaikh A. Solids suspension in stirred tanks with pitched blade turbines. Chem Eng Sci. 2003;58:2123-2140.

6. Kasat GR, Pandit AB. Review on mixing characteristics in solid-liquid and solid-liquid-gas reactor vessels. Can J Chem Eng. 2005;83:618-643.

7. Hosseini S, Patel D, Ein-Mozaffari F, Mehrvar M. Study of solid-liquid mixing in agitated tanks through electrical resistance tomography. Chem Eng Sci. 2010;65:1374-1384.

8. Carletti C, Montante G, Westerlund T, Paglianti A. Analysis of solid concentration distribution in dense solid-liquid stirred tanks by electrical resistance tomography. Chem Eng Sci. 2014;119:53-64.

9. Tahvildarian P, Ng H, D’Amato M, Drappel S, Ein-Mozaffari F, Upreti SR. Using electrical resistance tomography images to characterize the mixing of micron-sized polymeric particles in a slurry reactor. Chem Eng J. 2011;172:517-525.

10. Harrison ST, Stevenson R, Cilliers JJ. Assessing solids concentration homogeneity in Rushton-agitated slurry reactors using electrical resistance tomography (ERT). Chem Eng Sci. 2012;71:392-399.

11. Guida A, Fan X, Parker D, Nienow A, Barigou M. Positron emission particle tracking in a mechanically agitated solid-liquid suspension of coarse particles. Chem Eng Res Des. 2009;87:421-429. 
12. Guida A, Nienow AW, Barigou M. PEPT measurements of solid-liquid flow field and spatial phase distribution in concentrated monodisperse stirred suspensions. Chem Eng Sci. 2010;65:1905-1914.

13. Guha D, Ramachandran PA, Dudukovic MP. Flow field of suspended solids in a stirred tank reactor by Lagrangian tracking. Chem Eng Sci. 2007;62:6143-6154.

14. Tamburini A, Cipollina A, Micale G, Brucato A. Particle distribution in dilute solid liquid unbaffled tanks via a novel laser sheet and image analysis based technique. Chem Eng Sci. 2013;87:341-358.

15. Bittorf KJ, Kresta SM. Prediction of cloud height for solid suspensions in stirred tanks. Chem Eng Res Des. 2003;81:568-577.

16. Sardeshpande MV, Juvekar VA, Ranade VV. Solid suspension in stirred tanks: UVP measurements and CFD simulations. Can J Chem Eng. 2011;89:1112-1121.

17. Guiraud P, Costes J, Bertrand J. Local measurements of fluid and particle velocities in a stirred suspension. Chem Eng J. $1997 ; 68: 75-86$

18. Micheletti M, Yianneskis M. Study of fluid velocity characteristics in stirred solid-liquid suspensions with a refractive index matching technique. P I Mech Eng E-J Pro. 2004;218:191-204.

19. Virdung T, Rasmuson A. Measurements of continuous phase velocities in solid-liquid flow at elevated concentrations in a stirred vessel using LDV. Chem Eng Res Des. 2007;85:193-200.

20. Virdung T, Rasmuson A. Solid-liquid flow at dilute concentrations in an axially stirred vessel investigated using particle image velocimetry. Chem Eng Commun. 2007;195:18-34.

21. Unadkat H, Rielly CD, Hargrave GK, Nagy ZK. Application of fluorescent PIV and digital image analysis to measure turbulence properties of solid-liquid stirred suspensions. Chem Eng Res Des. 2009;87:573-586.

22. Gabriele A, Tsoligkas A, Kings I, Simmons M. Use of PIV to measure turbulence modulation in a high throughput stirred vessel with the addition of high Stokes number particles for both up-and down-pumping configurations. Chem Eng Sci. $2011 ; 66: 5862-5874$

23. Montante G, Paglianti A, Magelli F. Analysis of dilute solid-liquid suspensions in turbulent stirred tanks. Chem Eng Res Des. 2012;90:1448-1456.

24. Cui MM, Adrian RJ. Refractive index matching and marking methods for highly concentrated solid-liquid flows. Exp Fluids. $1997 ; 22: 261-264$.

25. Wiederseiner S, Andreini N, Epely-Chauvin G, Ancey C. Refractive-index and density matching in concentrated particle 
suspensions: a review. Exp Fluids. 2011;50:1183-1206.

26. Kohnen C, Bohnet M. Measurement and simulation of fluid flow in agitated solid/liquid suspensions. Chem Eng Technol. 2001;24:639-643.

27. Derksen JJ. Highly resolved simulations of solids suspension in a small mixing tank. AlChE J. 2012;58:3266-3278.

28. Mo J, Gao Z, Bao Y, Li Z, Derksen JJ. Suspending a solid sphere in laminar inertial liquid flow-experiments and simulations. AIChE J. 2015;61:1455-1469.

29. Derksen JJ. Simulations of solid-liquid scalar transfer for a spherical particle in laminar and turbulent flow. AIChE J. 2014;60:1202-1215.

30. Derksen JJ. Simulations of solid-liquid mass transfer in fixed and fluidized beds. Chem Eng J. 2014;255:233-244.

31. Malitson IH. Interspecimen comparison of the refractive index of fused silica. J Opt Soc Am. 1965;55:1205-1209.

32. Zachos A, Kaiser M, Merzkirch W. PIV measurements in multiphase flow with nominally high concentration of the solid phase. Exp Fluids. 1996;20:229-231.

33. Nouri J, Whitelaw J. Particle velocity characteristics of dilute to moderately dense suspension flows in stirred reactors. Int J Multiphase Flow. 1992;18:21-33.

34. Paul EL, Atiemo-Obeng VA, Kresta, SM. Handbook of industrial mixing: science and practice. New York: John Wiley \& Sons, Inc., 2004.

35. Christensen KT, Adrian RJ. Measurement of instantaneous Eulerian acceleration fields by particle image accelerometry: method and accuracy. Exp Fluids. 2002;33:759-769.

36. Christensen KT. The influence of peak-locking errors on turbulence statistics computed from PIV ensembles. Exp Fluids. 2004;36:484-497.

37. Willert C. The fully digital evaluation of photographic PIV recordings. Appl Sci Res. 1996;56: 79-102.

38. Hough PVC. Method and means for recognizing complex patterns. US patent 3,069,654,1962.

39. https://uk.mathworks.com/help/images/ref/imfindcircles.html. Accessed July 16, 2016.

40. Atherton TJ, Kerbyson DJ. Size invariant circle detection. Image Vision Comput.1999;17:795-803.

41. Feng Y, Goree J, Liu B. Errors in particle tracking velocimetry with high-speed cameras. Rev Sci Instrum. 2011;82:053707.

42. Khan F, Rielly C, Brown D. Angle-resolved stereo-PIV measurements close to a down-pumping pitched-blade turbine. Chem Eng Sci. 2006;61:2799-2806. 


\section{Table and Figure captions}

Table 1 Properties of continuous and disperse phase at $21^{\circ} \mathrm{C}$

Table 2 Effects of two-way coupling on the average flow.

Figure 1. Geometry of the stirred tank and the measurement plane, and definition of the coordinate system and impeller angle $\theta$ with respect to the measurement plane. In the view of the right panel, the impeller is rotating clockwise.

Figure 2. Images of a submerged glass sphere in (a) deionized water ( $n=1.3322)$; (b) olive oil ( $n=1.4672) ;(c)$ Phenyl silicone oil $(n=1.4610)$ at $\mathrm{T}=21^{\circ} \mathrm{C}$. The glass bead has $n=1.460$.

Figure 3. Effect of the temperature on properties of phenyl silicone oil: (a) refractive index; (b) dynamic viscosity. In the fitted equations, $\mathrm{T}$ is in ${ }^{\circ} \mathrm{C}, \mu$ in $\mathrm{Pa} \cdot \mathrm{S}$.

Figure 4. Images of the tank in operation with $8 \%$ solids by volume. (a) \& (c): With refractive index matching (silica glass spheres in phenyl silicone oil); (b) \& (d) without refractive index matching (soda-lime glass spheres in phenyl silicone oil). Figure 5. Results of boundary detections: (a) Original PIV image; (b) Few particles are detected with 'Sensitivity' value equal to 0.88; (c) Many error detections with 'Sensitivity' value equal to 0.945; (d) Final detection results with Sensitivity' value equal to 0.925 .

Figure 6. (a) Original PIV image; (b) PIV images after boundary detections; (c) instantaneous local velocity field; (d) instantaneous local velocity field without vectors inside the particles.

Figure 7. Comparisons of instantaneous velocity field in different locations of the stirred tank at impeller angle $\theta=60^{\circ}$ : Left part of each sub-figure: single-phase system; right: solid-liquid system (tank-averaged solids volume fraction is $8 \%$ ). The locations of the panels in the tank are indicated at the top; the reference vector applies to all PIV images.

Figure 8. Comparison of instantaneous velocity field at impeller angle $\theta=60^{\circ}$ (a) single-phase system; (b) solid-liquid two phase system with solids volume fraction of $8 \%$. The resolution of the experiment is three times as high in each direction as the density of the velocity vectors in this figure.

Figure 9. Averaged solids volume fraction contours at impeller angle $\theta=60^{\circ}$ with different particle volumetric concentrations: (a) $1 \%$; (b) $3 \%$; (c) $5 \%$; (d) $8 \%$.

Figure 10. Averaged solids volume fraction contours at different impeller angles: (a) $\theta=0^{\circ}$; (b) $\theta=30^{\circ}$.

Figure 11. Vertical profiles of average solids volume fraction with impeller angle $\theta=60^{\circ}$ at two horizontal positions: (a) $\mathrm{x} / \mathrm{T}=0.20 ;$ (b) $\mathrm{x} / \mathrm{T}=0.47$.

Figure 12. Vertical profiles of average solids volume fraction for overall solids volume fraction $\Phi=5 \%$ with different impeller angles at two horizontal positions: (a) $x / T=0.20$; (b) $x / T=0.47$.

Figure 13. Averaged vertical positions of the particles in the imaging plane as a function of the number of images involved in the averaging process. (a) For different overall solids volume fraction at impeller angle $\theta=60^{\circ}$; (b) for different impeller angles at an overall solids volume fraction $\Phi=5 \%$.

Figure 14. Normalized impeller-angle-resolved mean velocity flow field of the continuous phase for different solids volume fraction: Panel (a): 0\%; Panel (b): $1 \%$; Panel (c): $5 \%$ and the impeller angle $0^{\circ}, 30^{\circ}, 60^{\circ}$ from the left to right; Panel (d): $8 \%$ with impeller angle $\theta=60^{\circ}$. The resolution of the experiment is three times as high in each direction as the density of the velocity vectors in the figure.

Figure 15. (a) Vertical profile of normalized mean radial velocity at $x / T=0.45$ with impeller angle $\theta=60^{\circ}$; (b) Horizontal profile of normalized mean axial velocity at $\mathrm{z} / \mathrm{H}=0.25$ with impeller angle $\theta=60^{\circ}$.

Figure 16. Normalized turbulent kinetic energy contours for different solids volume fraction: Panel (a): 0\%; Panel (b): $1 \%$; Panel (c): $5 \%$ and the impeller angle is $0^{\circ}, 30^{\circ}, 60^{\circ}$ from left to right; Panel (d): $8 \%$ for impeller angle $\theta=60^{\circ}$.

Figure 17. (a) Vertical profile of normalized radial $\mathrm{rms}$ velocity at $x / T=0.45$ with impeller angle $\theta=60^{\circ}$; (b) $\mathrm{Horizontal}$ profile of normalized axial rms velocity at $\mathrm{z} / \mathrm{H}=0.20$ with impeller angle $\theta=60^{\circ}$. 
Table 1

Table 1 Properties of continuous and disperse phase at $21^{\circ} \mathrm{C}$.

\begin{tabular}{ccc}
\hline & phenyl silicone oil & silica glass spheres \\
\hline Density $\left(\mathrm{kg} \mathrm{m}^{-3}\right)$ & 991 & 2210 \\
Dynamic viscosity (Pa.s) & 0.02087 & \\
Refractive index & 1.461 & 1.460 \\
\hline
\end{tabular}

Table 2

Table 2 Effects of two-way coupling on the average flow.

\begin{tabular}{cc}
\hline solids volume fraction & $U_{a v} / \mathrm{v}_{\text {tip }}$ \\
\hline $0 \%$ & 0.19 \\
$1 \%$ & 0.184 \\
$5 \%$ & 0.135 \\
\hline
\end{tabular}


Figure 1
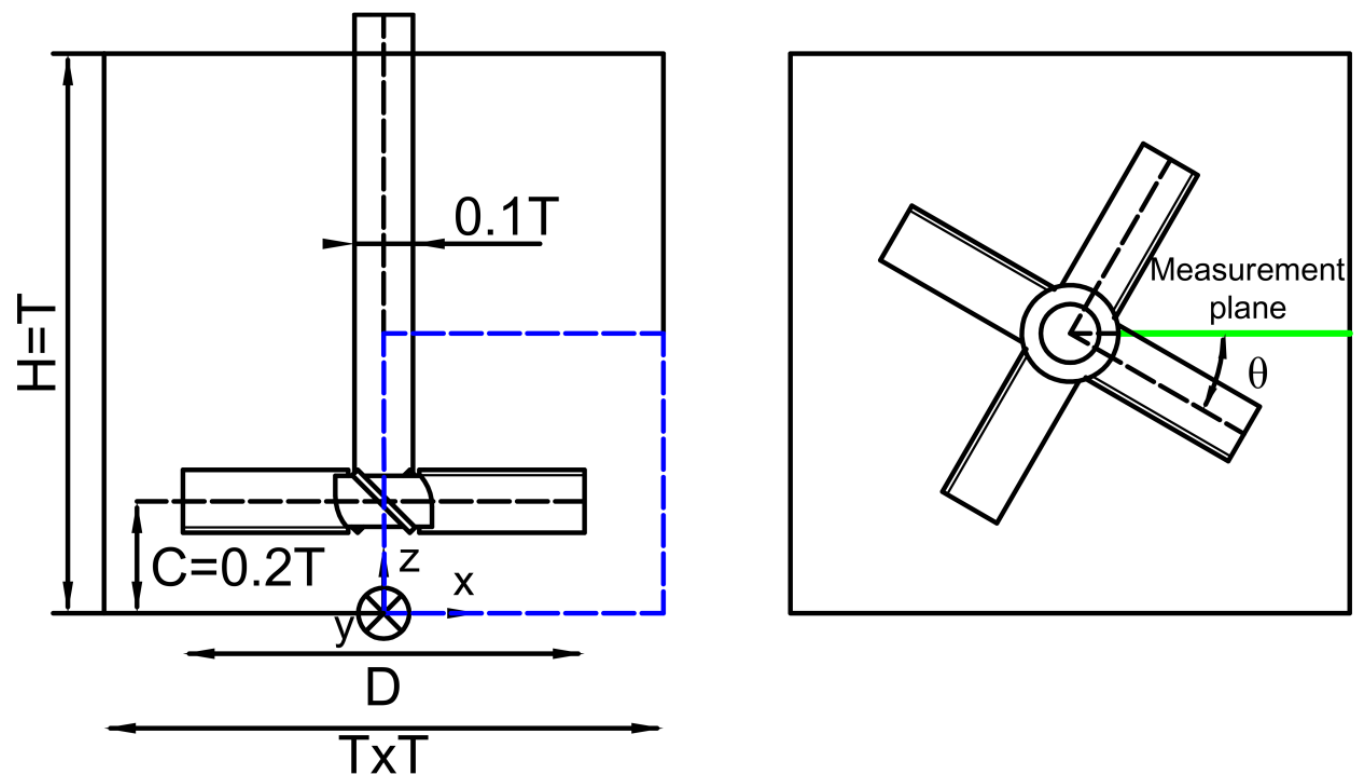

Figure 1. Geometry of the stirred tank and the measurement plane, and definition of the coordinate system and impeller angle $\theta$ with respect to the measurement plane. In the view of the right panel, the impeller is rotating clockwise. 


\section{Figure 2}

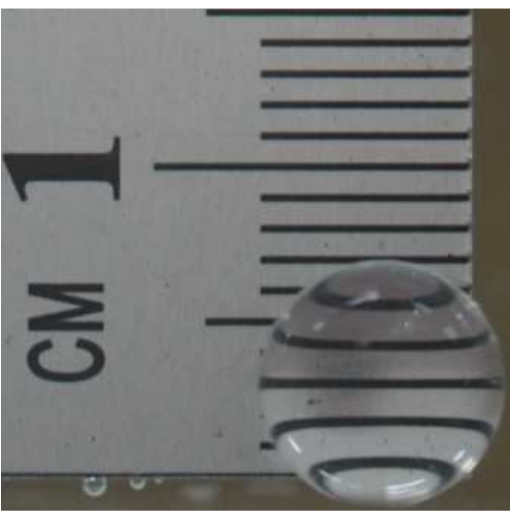

(a)

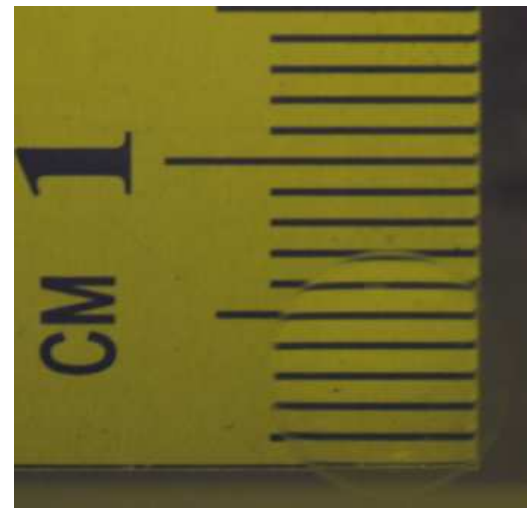

(b)

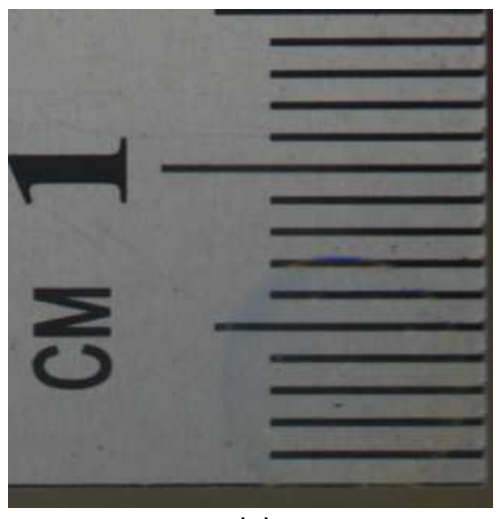

(c)

Figure 2. Images of a submerged glass sphere in (a) deionized water ( $n=1.3322)$; (b) olive oil ( $n=1.4672)$; (c) Phenyl silicone oil $(n=1.4610)$ at $\mathrm{T}=21^{\circ} \mathrm{C}$. The glass bead has $n=1.460$. 


\section{Figure 3}

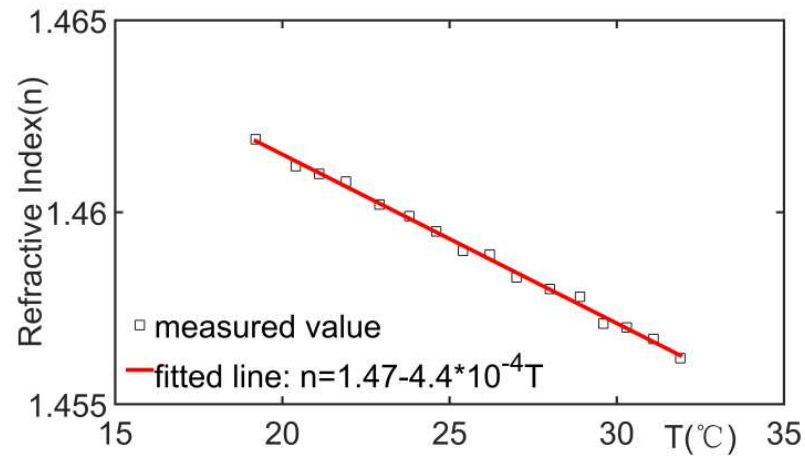

(a)

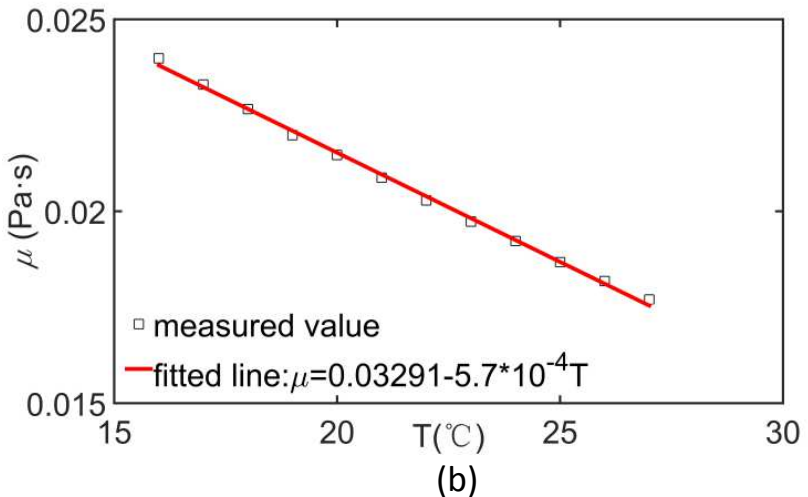

(b)

Figure 3. Effect of the temperature on properties of phenyl silicone oil:

(a) refractive index; (b) dynamic viscosity. In the fitted equations, $\mathrm{T}$ is in ${ }^{\circ} \mathrm{C}, \mu$ in Pa.s. 


\section{Figure 4}
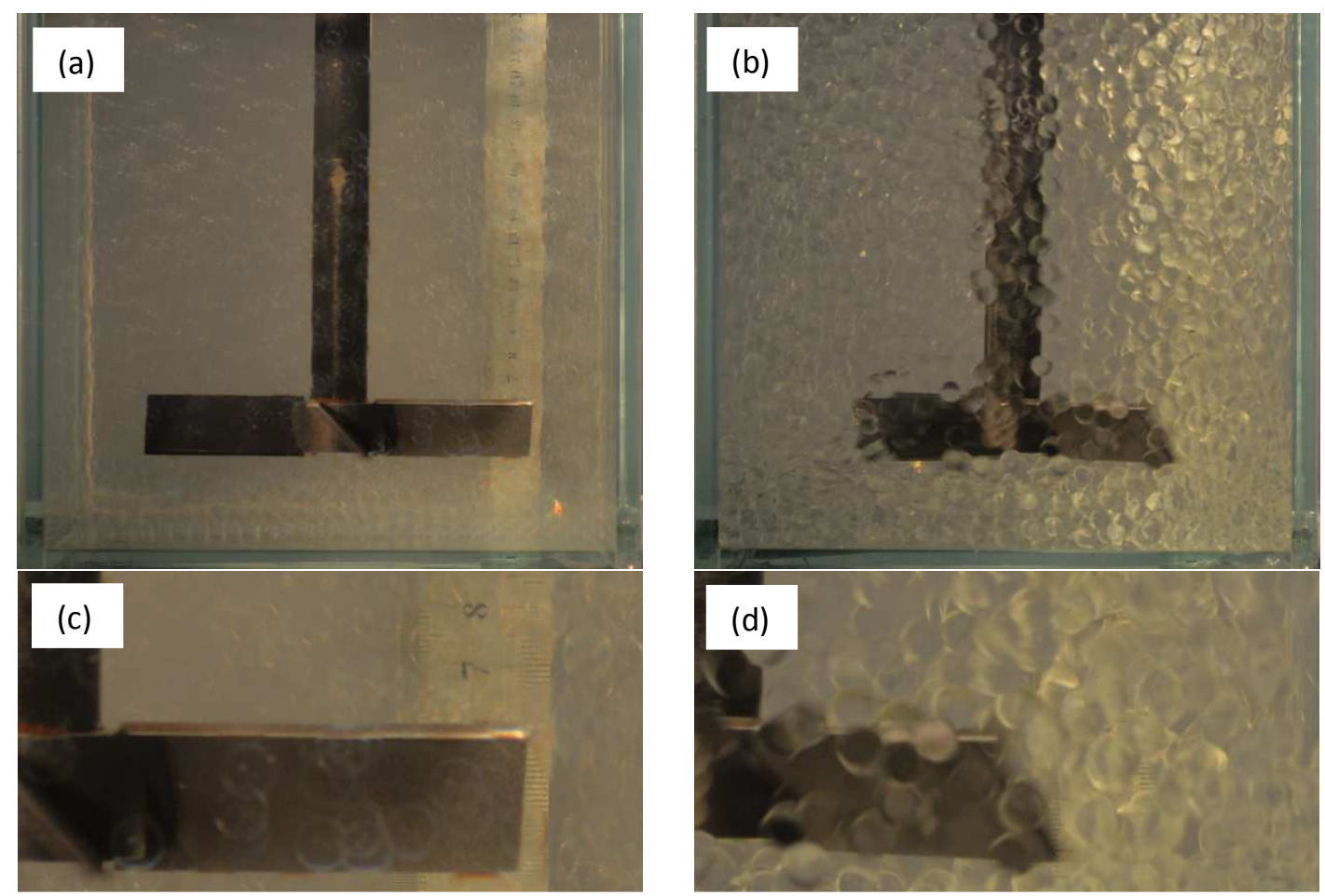

Figure 4. Images of the tank in operation with $8 \%$ solids by volume. (a) \& (c): With refractive index matching (silica glass spheres in phenyl silicone oil); (b) \& (d) without refractive index matching (soda-lime glass spheres in phenyl silicone oil). 


\section{Figure 5}

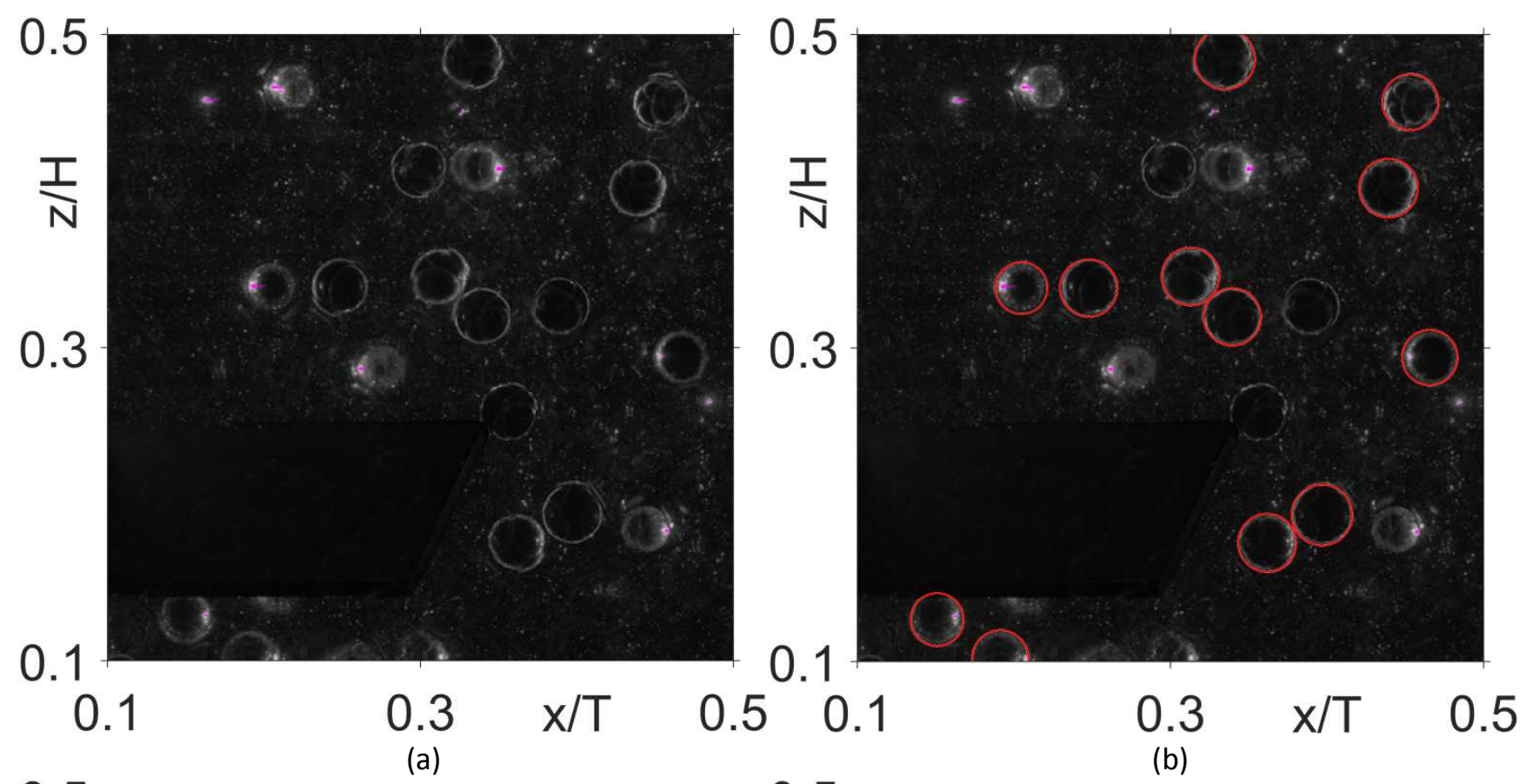

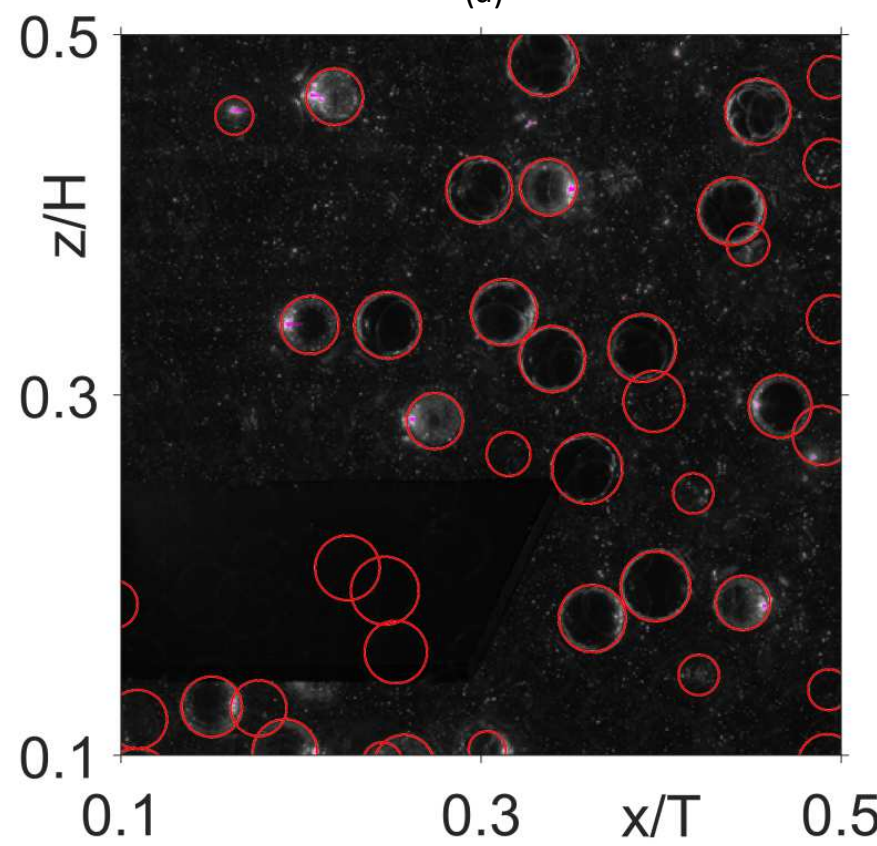

(c)

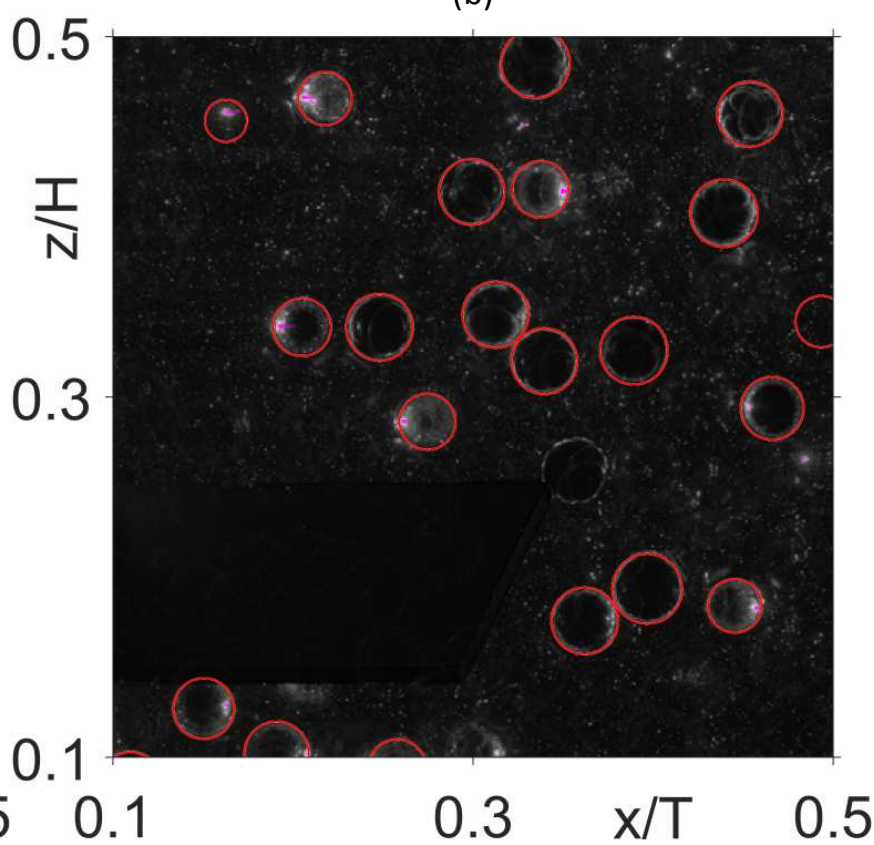

(d)

Figure 5. Results of boundary detections: (a) Original PIV image; (b) Few particles are detected with 'Sensitivity' value equal to 0.88 ; (c) Many error detections with 'Sensitivity' value equal to 0.945 ; (d) Final detection results with 'Sensitivity' value equal to 0.925 . 


\section{Figure 6}

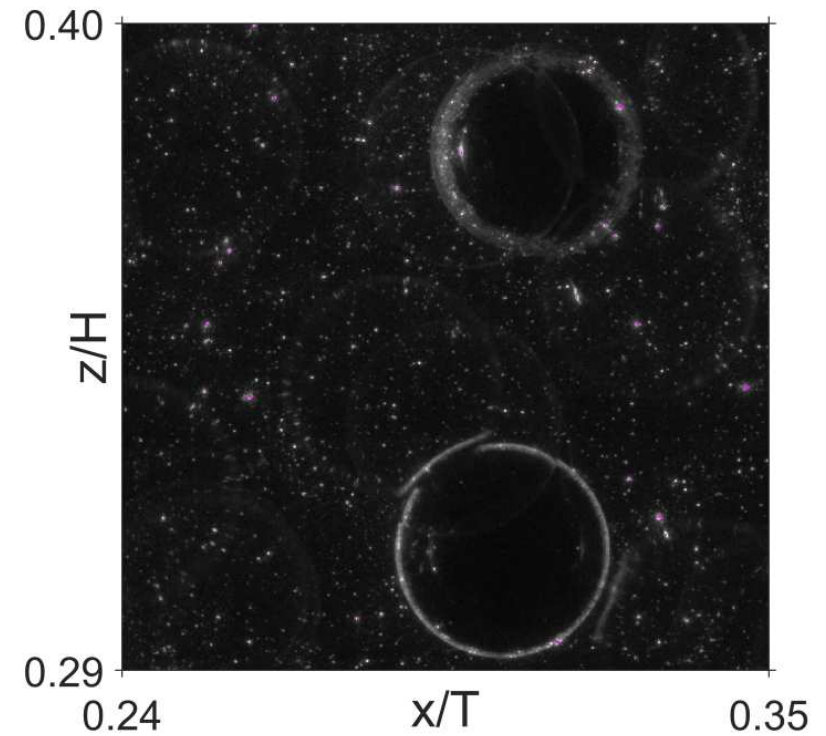

(a)

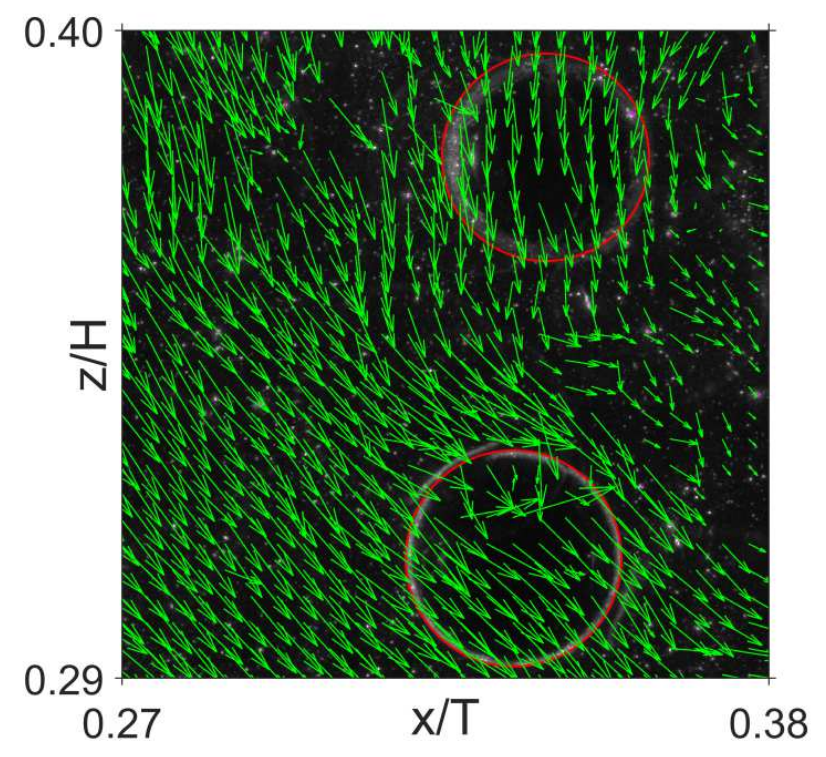

(c)

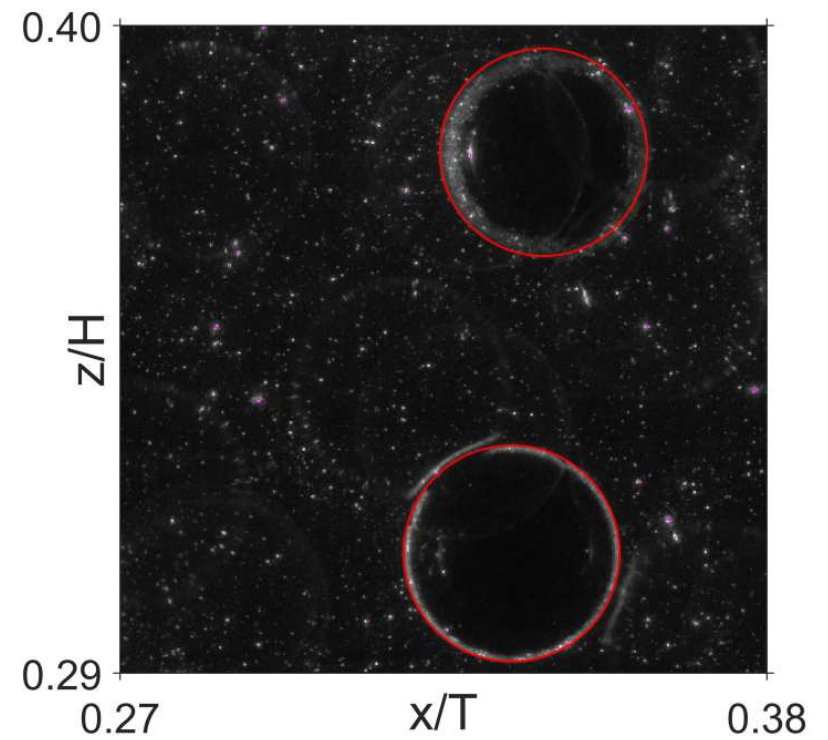

(b)

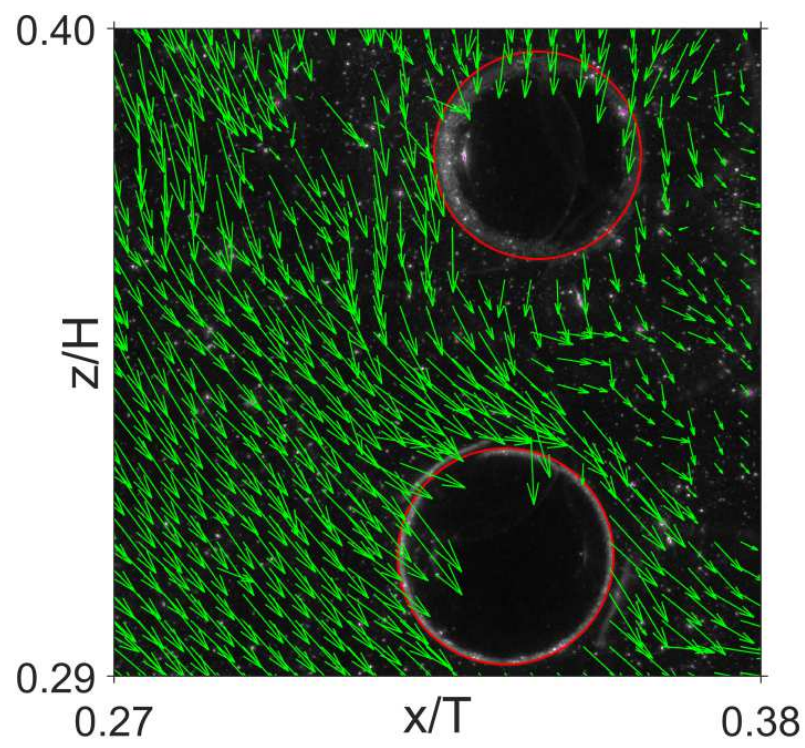

(d)

Figure 6. (a) Original PIV image; (b) PIV images after boundary detections;

(c) instantaneous local velocity field; (d) instantaneous local velocity field without vectors inside the particles. 


\section{Figure 7}
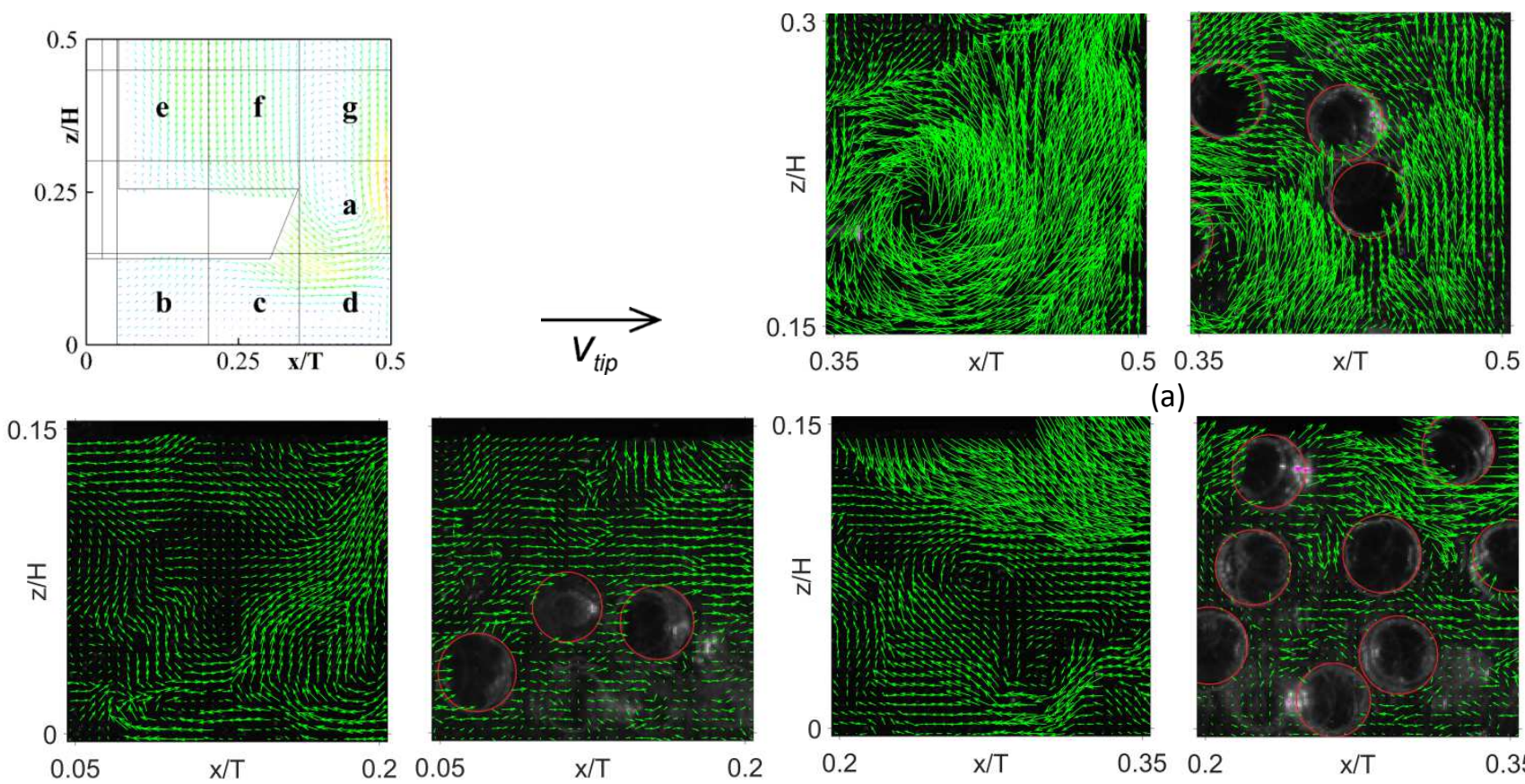

(b)
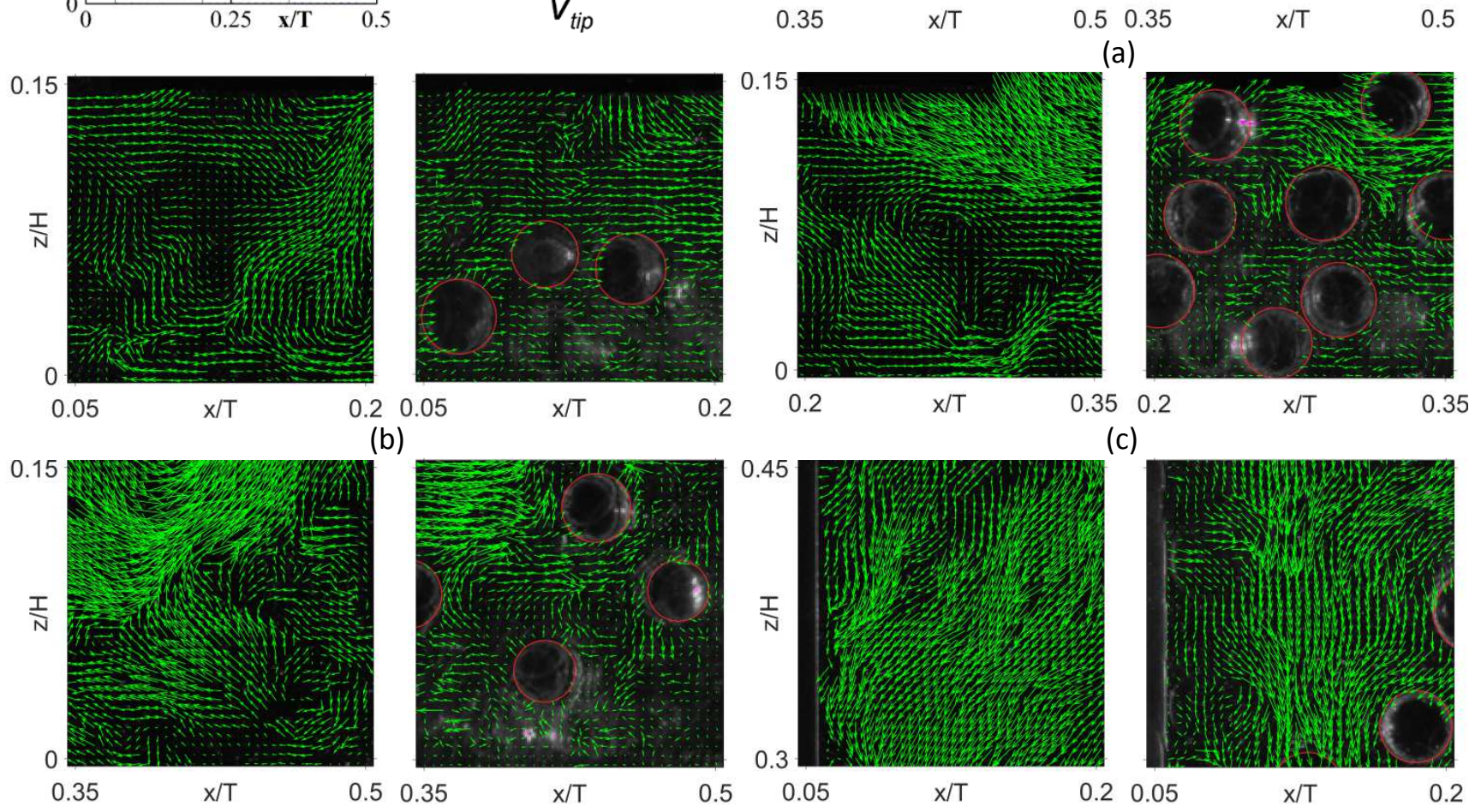

(c)

(d)
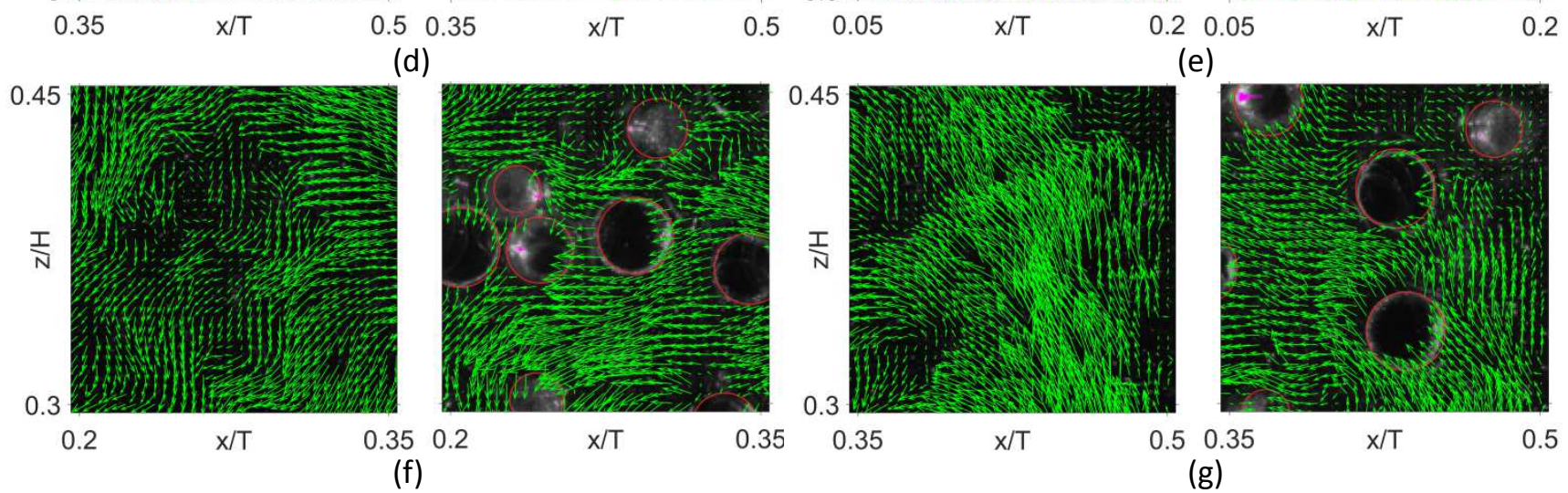

Figure 7. Comparisons of instantaneous velocity field in different locations of the stirred tank at impeller angle $\theta=60^{\circ}$ : Left part of each sub-figure: single-phase system; right: solid-liquid system (tank-averaged solids volume fraction is $8 \%$ ). The locations of the panels in the tank are indicated at the top; the reference vector applies to all PIV images. 


\section{Figure 8}

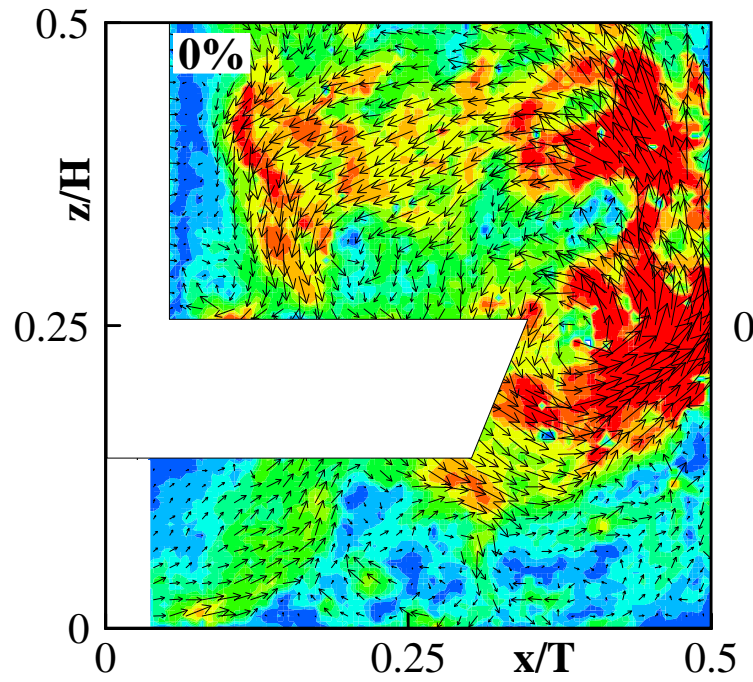

(a)

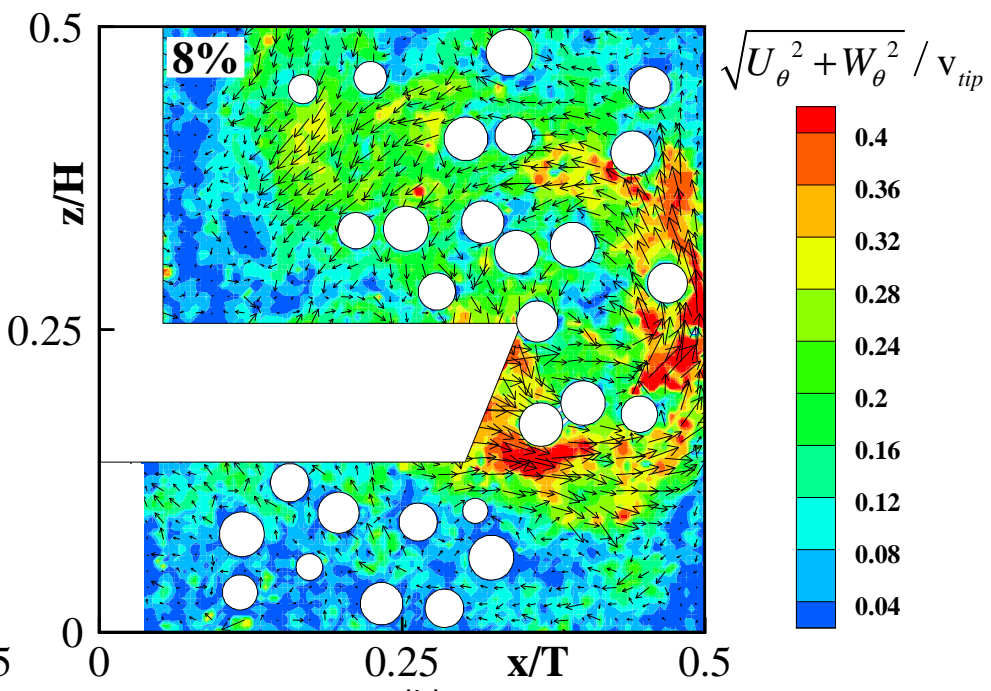

(b)

Figure 8. Comparison of instantaneous velocity field at impeller angle $\theta=60^{\circ}$ (a) single-phase system; (b) solid-liquid two phase system with solids volume fraction of $8 \%$. The resolution of the experiment is three times as high in each direction as the density of the velocity vectors in this figure. 


\section{Figure 9}

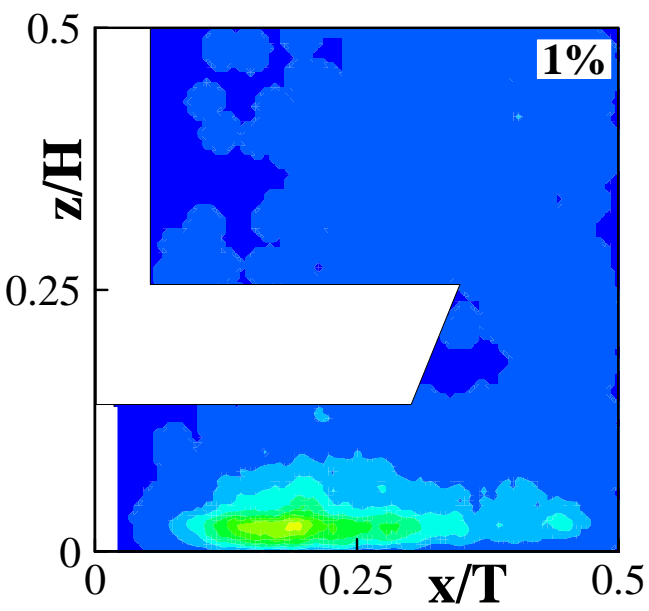

(a)

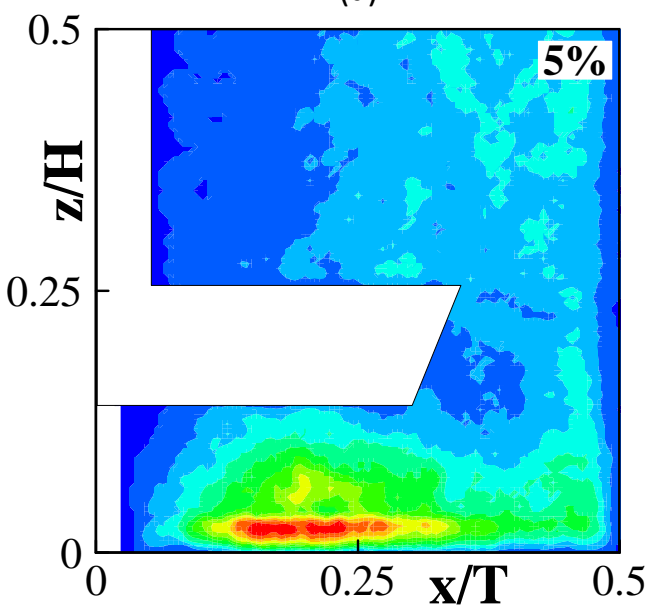

(c)

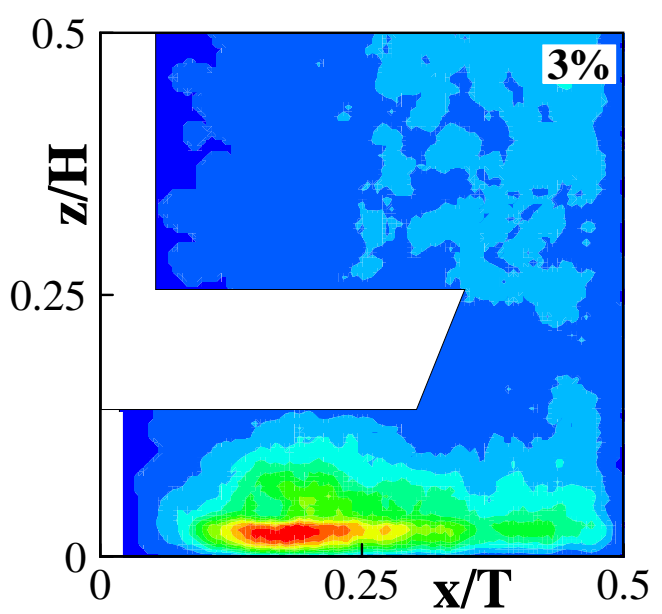

(b)

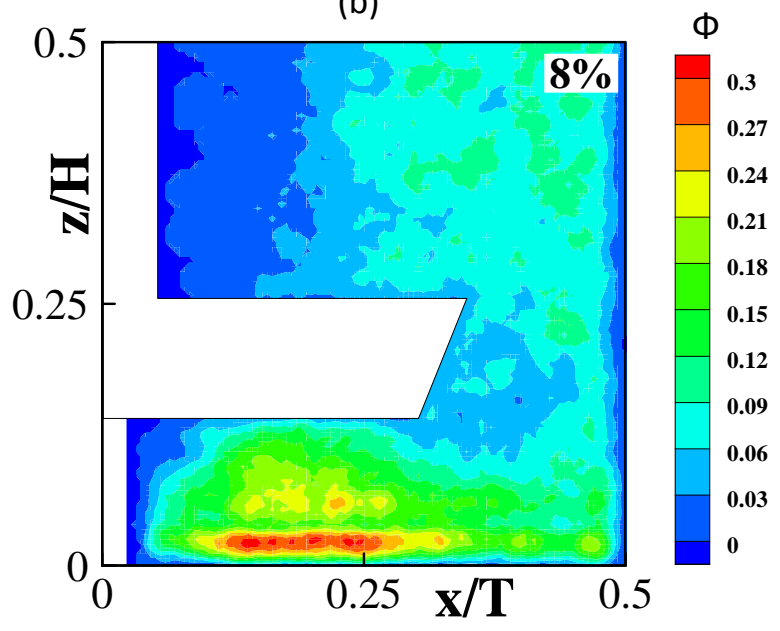

(d)

Figure 9. Averaged solids volume fraction contours at impeller angle $\theta=60^{\circ}$ with different particle volumetric concentrations: (a)1\%; (b) $3 \%$; (c) $5 \%$; (d) $8 \%$. 
Figure 10

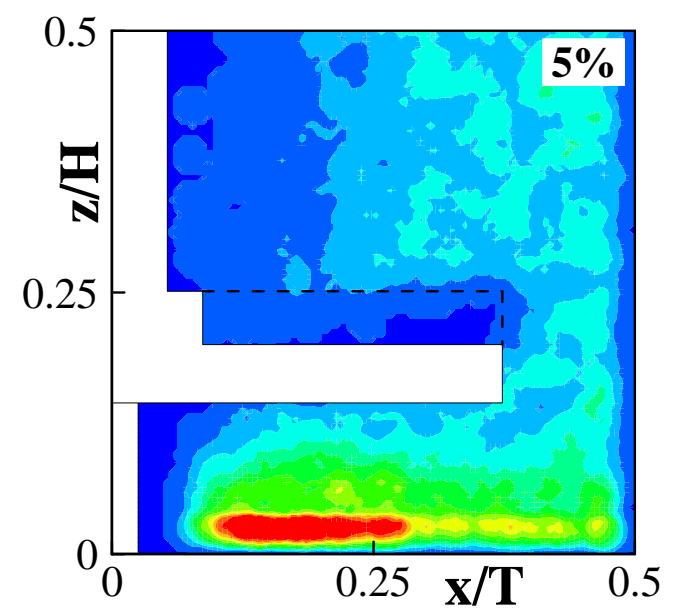

(a)

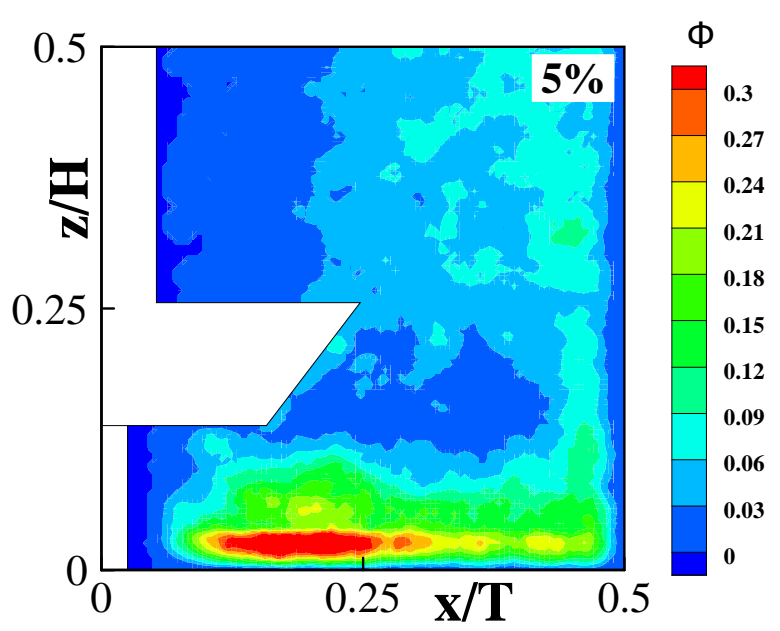

(b)

Figure 10. Averaged solids volume fraction contours at different impeller angles: (a) $\theta=0^{\circ}$; (b) $\theta=30^{\circ}$. 
Figure 11
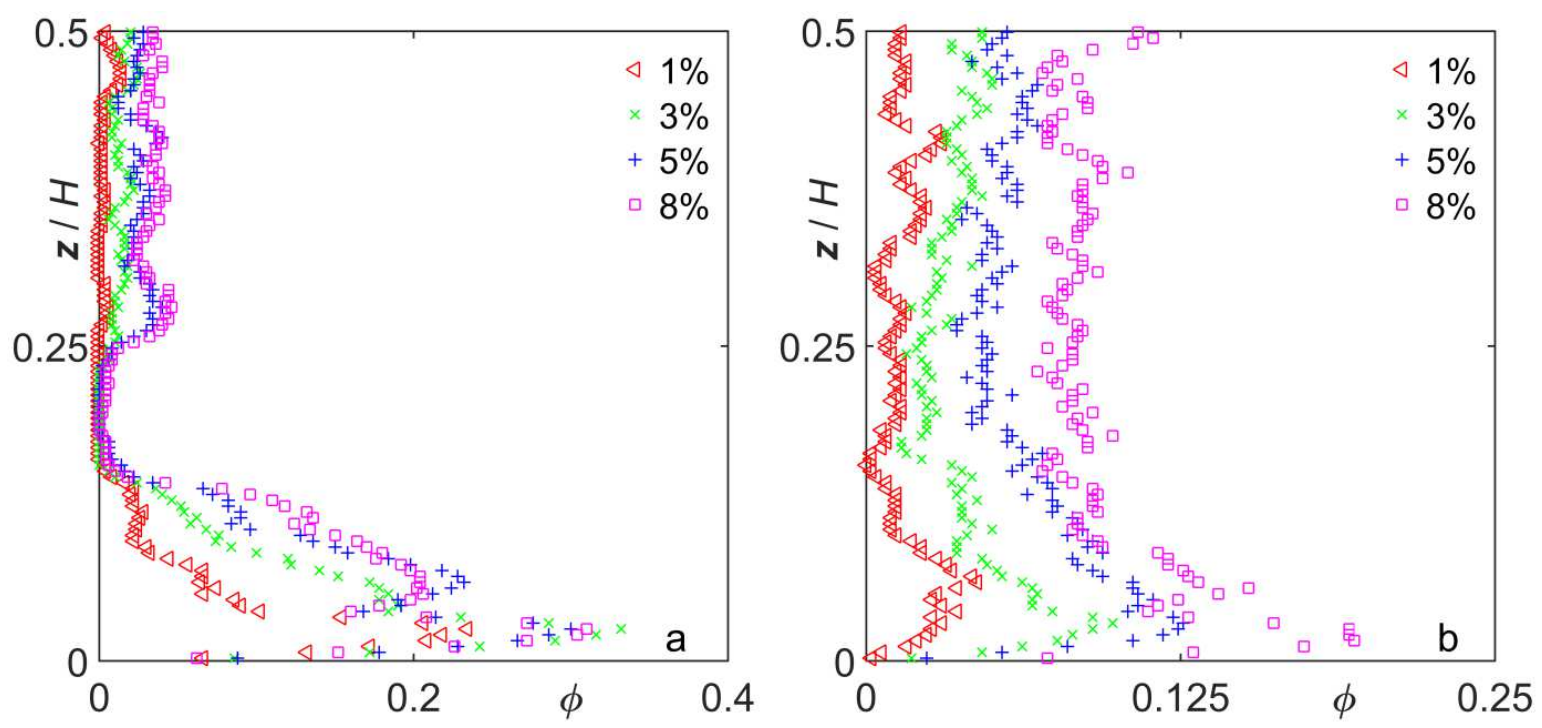

Figure 11. Vertical profiles of average solids volume fraction with impeller angle $\theta=60^{\circ}$ at two horizontal positions: (a) $x / T=0.20 ;$ (b) $x / T=0.47$. 
Figure 12
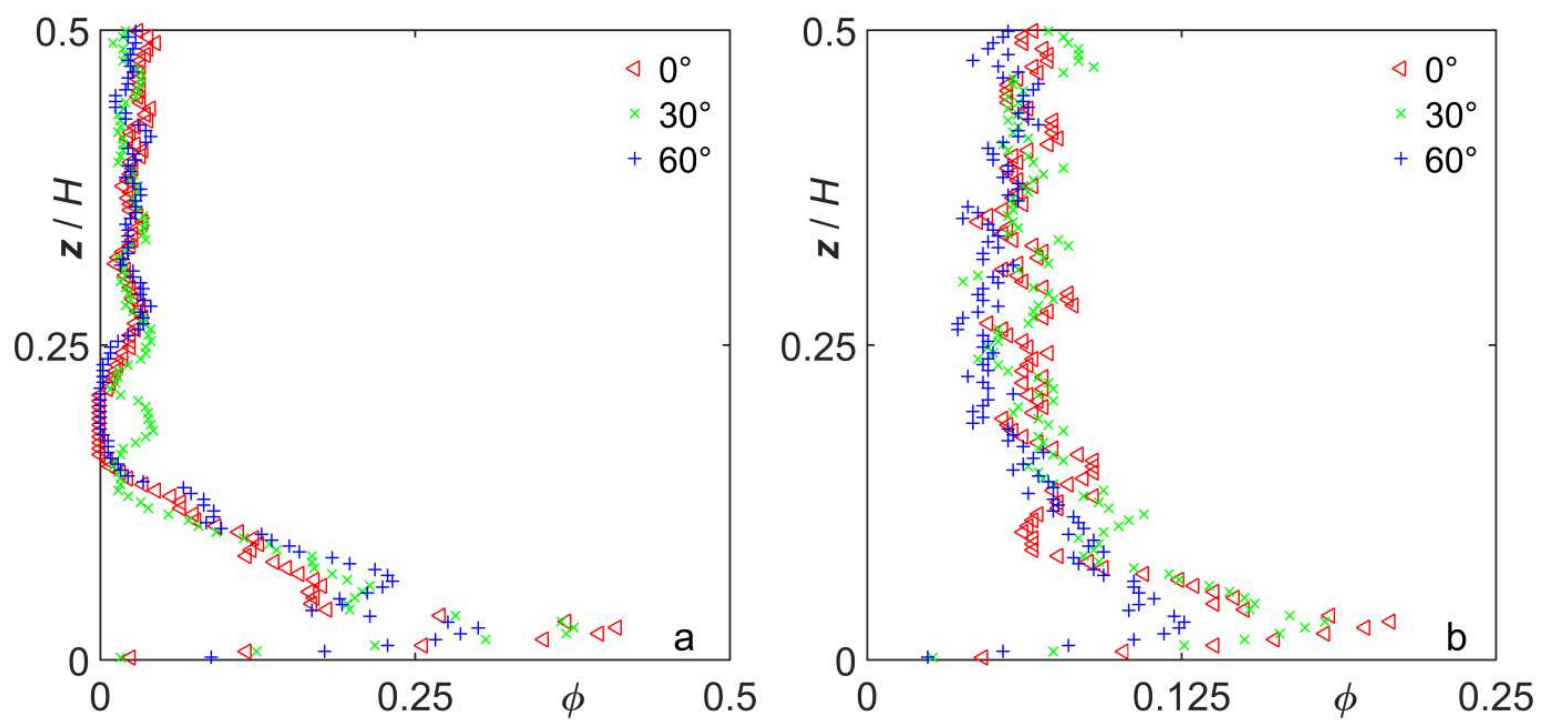

Figure 12. Vertical profiles of average solids volume fraction for overall solids volume fraction $\Phi=5 \%$ with different impeller angles at two horizontal positions: (a) $x / T=0.20 ;(b) x / T=0.47$. 


\section{Figure 13}
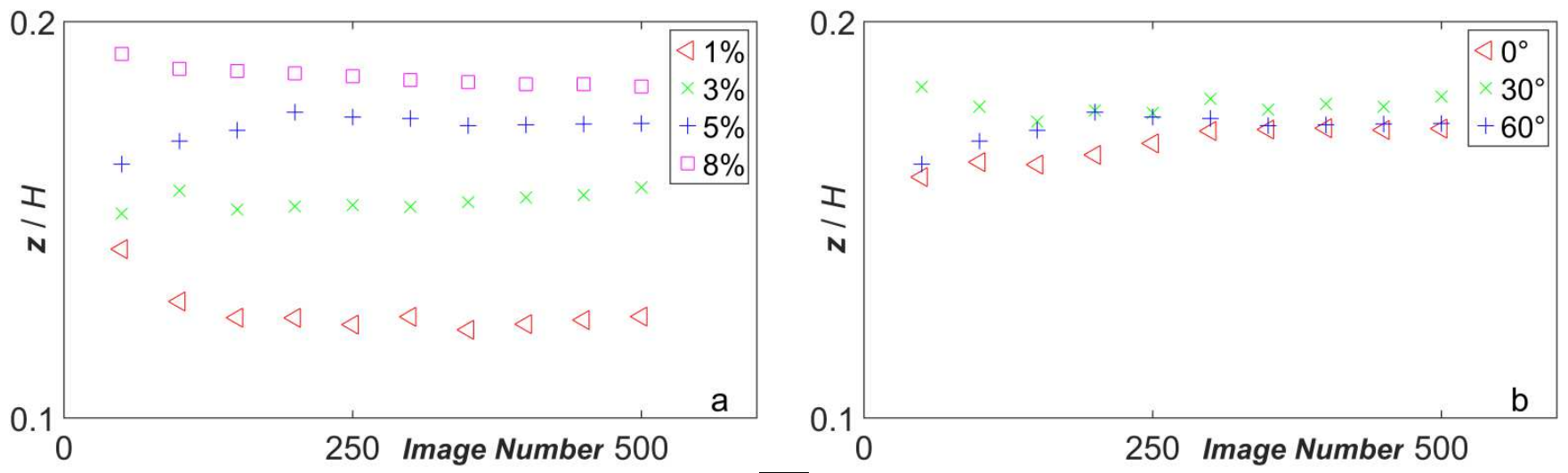

Figure 13. Averaged vertical positions of the particles in the imaging plane as a function of the number of images involved in the averaging process. (a) For different overall solids volume fraction at impeller angle $\theta=60^{\circ}$; (b) for different impeller angles at an overall solids volume fraction $\Phi=5 \%$. 
Figure 14
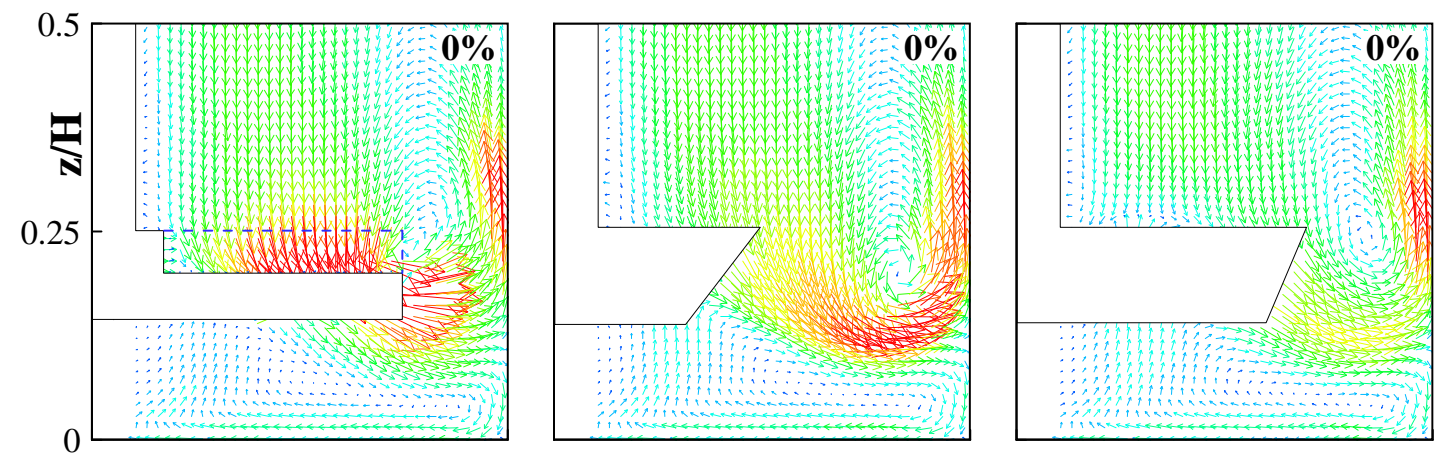

(a)
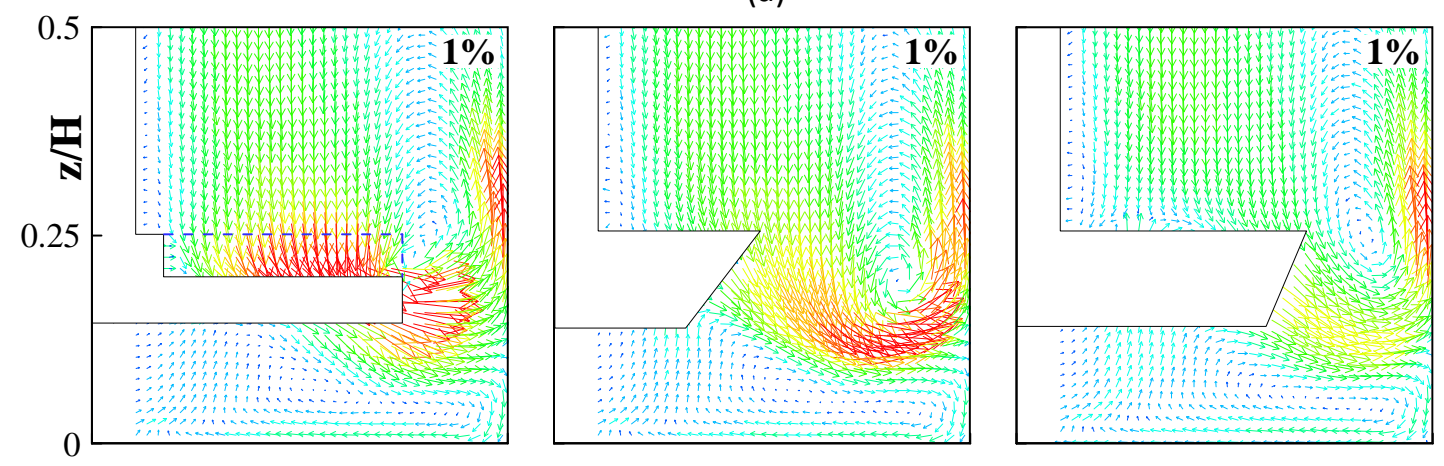

(b)
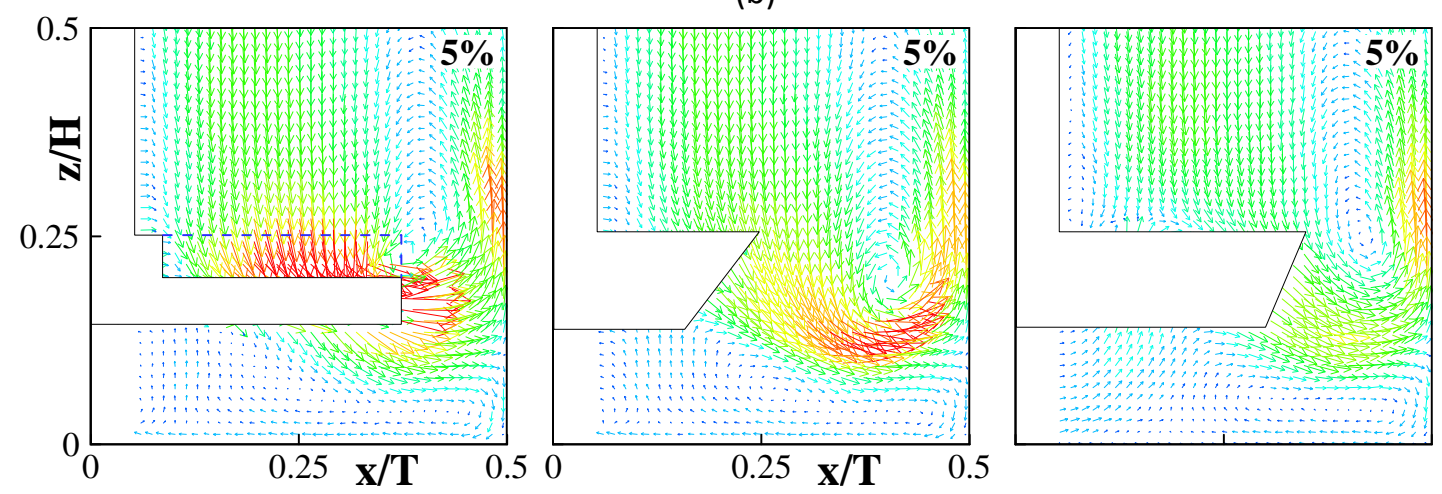

$\sqrt{\bar{U}_{\theta}^{2}+\bar{W}_{\theta}^{2}} / \mathrm{v}_{t i p}$

(c)

\begin{tabular}{|l|l}
0.4 \\
0.36 \\
0.32 \\
0.28 \\
0.24 \\
0.2 \\
0.16 \\
0.12 \\
0.08 \\
0.04 \\
0
\end{tabular}

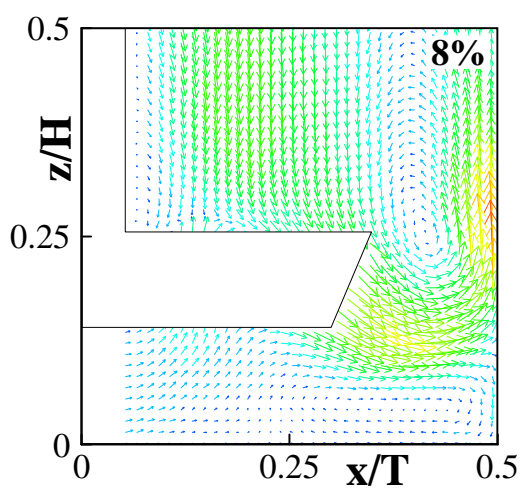

(d)

Figure 14. Normalized impeller-angle-resolved mean velocity flow field of the continuous phase for different solids volume fraction: Panel (a): $0 \%$; Panel (b): $1 \%$; Panel (c): $5 \%$ and the impeller angle $0^{\circ}, 30^{\circ}, 60^{\circ}$ from the left to right; Panel (d): $8 \%$ with impeller angle $\theta=60^{\circ}$. The resolution of the experiment is three times as high in each direction as the density of the velocity vectors in the figure. 
Figure 15
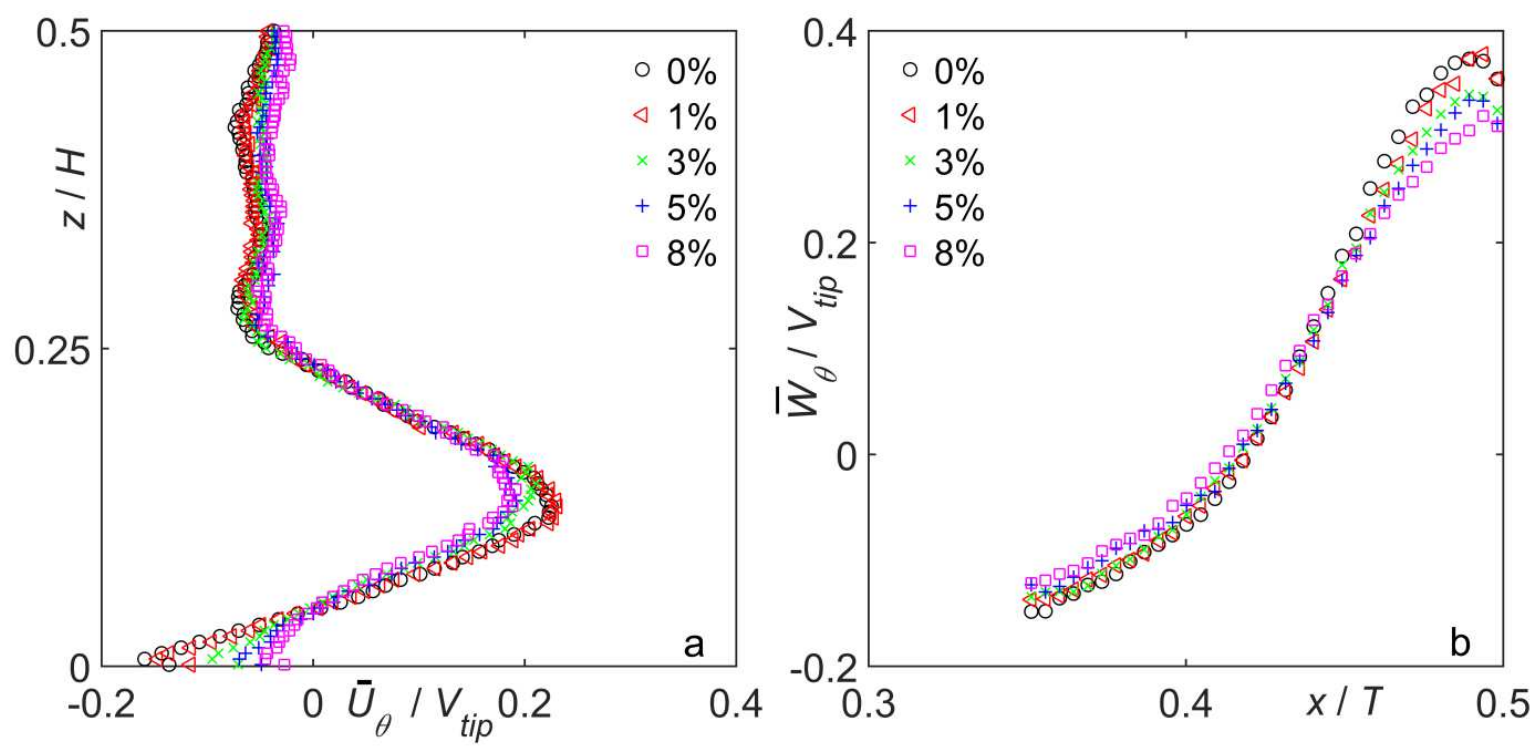

Figure 15. (a) Vertical profile of normalized mean radial velocity at $x / T=0.45$ with impeller angle $\theta=60^{\circ}$; (b) Horizontal profile of normalized mean axial velocity at $\mathrm{z} / \mathrm{H}=0.25$ with impeller angle $\theta=60^{\circ}$. 


\section{Figure 16}
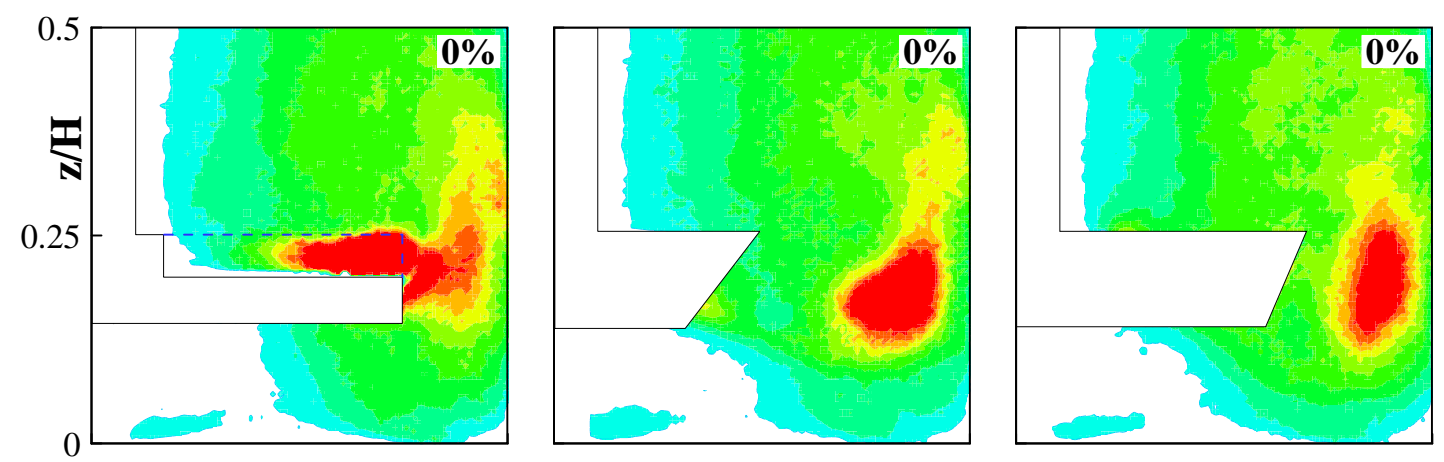

(a)
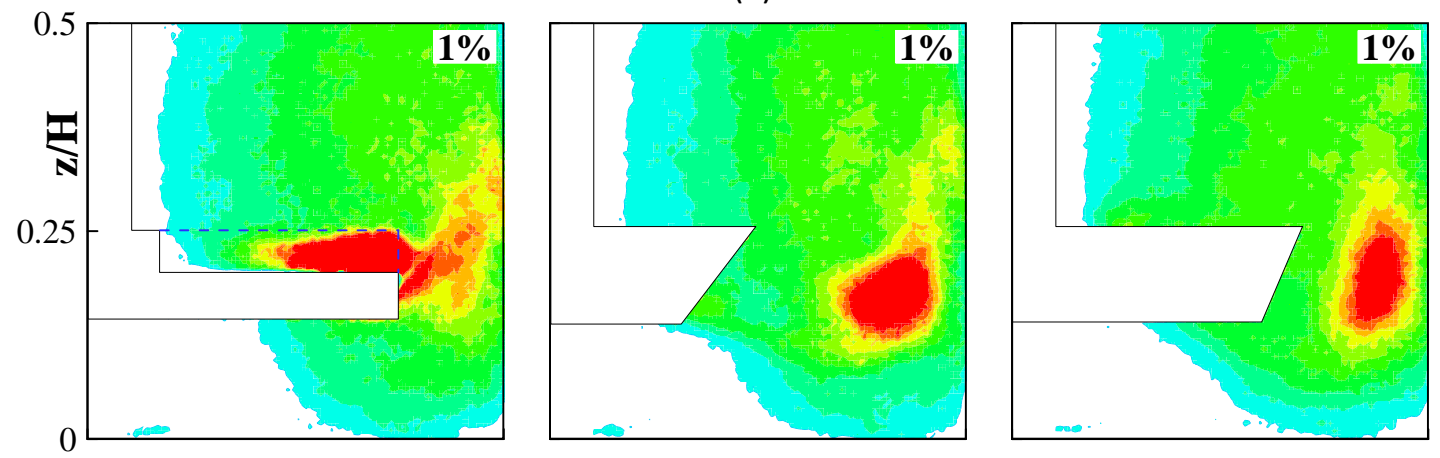

(b)
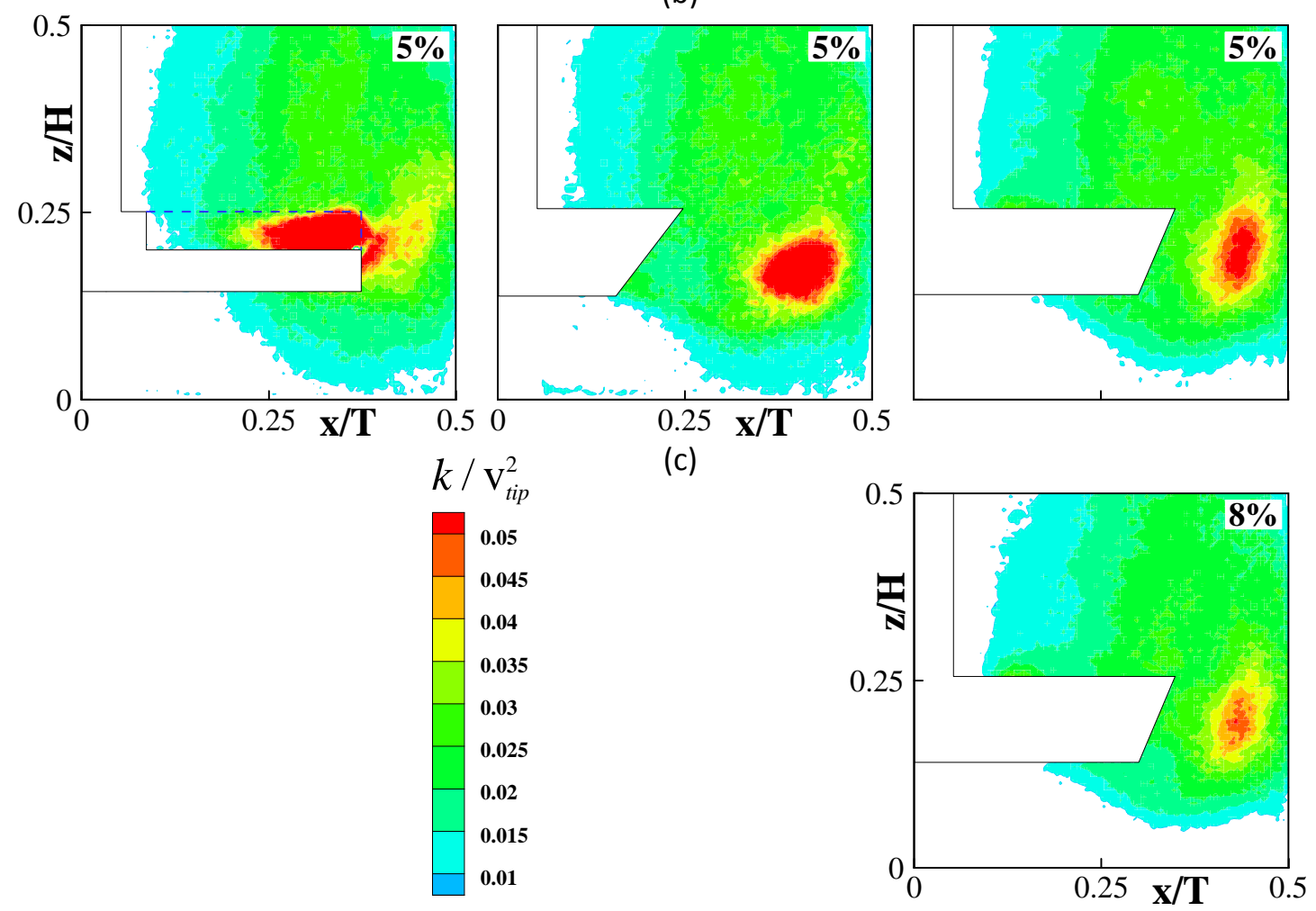

(d)

Figure 16. Normalized turbulent kinetic energy contours for different solids volume fraction: Panel (a): $0 \%$; Panel (b): $1 \%$; Panel (c): $5 \%$ and the impeller angle is $0^{\circ}, 30^{\circ}, 60^{\circ}$ from left to right; Panel (d): $8 \%$ for impeller angle $\theta=60^{\circ}$. 
Figure 17
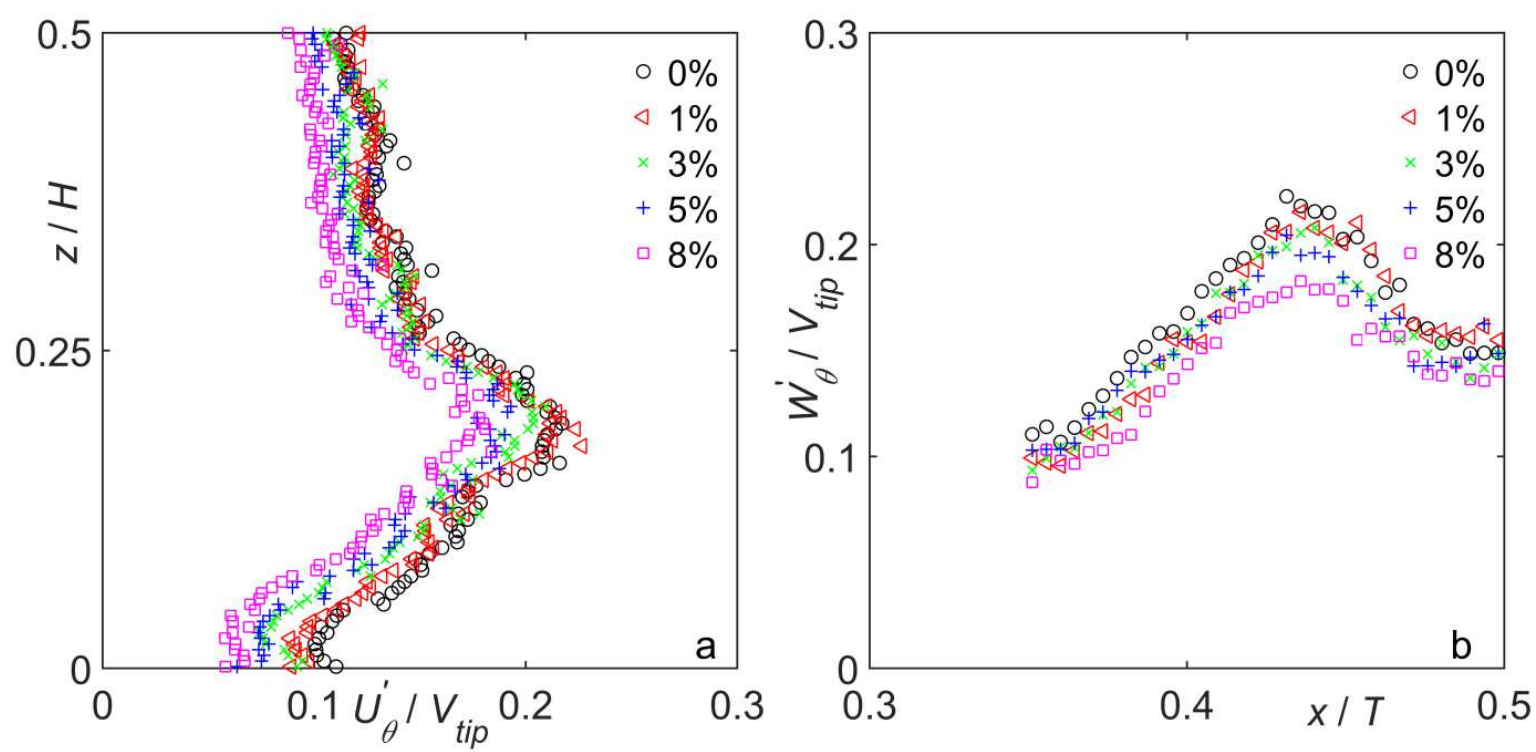

Figure 17. (a) Vertical profile of normalized radial rms velocity at $x / T=0.45$ with impeller angle $\theta=60^{\circ}$; (b) Horizontal profile of normalized axial rms velocity at $\mathrm{z} / \mathrm{H}=0.20$ with impeller angle $\theta=60^{\circ}$. 